\title{
High resolution near-infrared spectro-imaging of NGC $7027^{\star}$
}

\author{
P. Cox ${ }^{1}$, P. J. Huggins ${ }^{2}$, J.-P. Maillard ${ }^{3}$, E. Habart ${ }^{1}$, C. Morisset ${ }^{4}$, R. Bachiller ${ }^{5}$, and T. Forveille ${ }^{6}$ \\ 1 Institut d'Astrophysique Spatiale, Bât. 121, Université de Paris XI, 91405 Orsay, France \\ 2 Physics Department, New York University, 4 Washington Place, New York, NY 10003, USA \\ 3 Institut d'Astrophysique de Paris, CNRS, 98b bd. Arago, 75014 Paris, France \\ ${ }^{4}$ Institut d'Astrophysique de Marseille, CNRS \& Univ. de Provence, BP 8, 13376 Marseille Cedex 12, France \\ 5 Observatorio Astronómico Nacional (IGN), Apt 1143, 28800 Alcalá de Henares, Spain \\ 6 Observatoire de Grenoble, BP 53X, 38041 Grenoble Cedex, France
}

Received 7 August 2001 / Accepted 5 December 2001

\begin{abstract}
We present near-infrared spectro-imaging of the young planetary nebula NGC 7027 between 2.10 and $2.20 \mu \mathrm{m}$ with high spatial $\left(0.5^{\prime \prime}\right)$ and high spectral $\left(8.7 \mathrm{~km} \mathrm{~s}^{-1}\right)$ resolution. The observations, made using $B E A R$ at the CFHT, reveal the detailed morphology and kinematics of the ionized nebula (in the Br $\gamma$ line and 16 other atomic lines) and the surrounding molecular envelope (in the 1-0 S(1) line of $\mathrm{H}_{2}$ ). The observations show that the ionized gas forms an elongated $\left(\sim 6^{\prime \prime} \times 12^{\prime \prime}, \mathrm{PA}=32^{\circ}\right)$, limb-brightened shell with an expansion velocity of $19.5 \mathrm{~km} \mathrm{~s}^{-1}$ along the line of sight. The shell is composed of numerous small condensations and has nearly parallel sides with flattened ends that are not well matched by a uniform ellipsoidal model. Low level $\mathrm{Br} \gamma$ emission is detected at high red- and blue-shifted velocities $\left( \pm 55 \mathrm{~km} \mathrm{~s}^{-1}\right)$ along a bipolar axis at $\mathrm{PA}=60^{\circ}$ that deviates significantly from that of the main nebula. The $\mathrm{H}_{2}$ emission is distributed at the periphery of the ionized gas, in a limb-brightened, bi-conical shell $\left(\sim 10^{\prime \prime} \times 13^{\prime \prime}, \mathrm{PA}=28^{\circ}\right)$ with enhanced emission at the equator and complex structure at the ends of the major axis. The $\mathrm{H}_{2}$ emission traces the inner edge of the extended molecular envelope seen in $\mathrm{CO}$, and its distribution and intensity are well matched by model predictions of a high-density $\left(n_{\mathrm{H}} \sim 10^{6}-10^{5} \mathrm{~cm}^{-3}\right)$ photo-dissociation region. The kinematic structure of the $\mathrm{H}_{2}$ emission reveals a remarkable series of lobes and openings in the molecular shell. These features are point symmetric about the center, and the most prominent pair aligns with the high velocity, bipolar emission seen in $\operatorname{Br} \gamma$. These observations demonstrate recent activity by collimated outflows in NGC 7027, with a multiple, bipolar geometry. The interaction of the outflows with the surrounding envelope has significantly affected the morphology of the developing nebula and its environment, and their presence in this well-studied archetype underscores the general importance of outflows in the early shaping history of planetary nebulae.
\end{abstract}

Key words. infrared: ISM - ISM: lines and bands - ISM: molecules - planetary nebulae: general planetary nebulae: individual: NGC 7027

\section{Introduction}

The circumstellar envelopes of asymptotic giant branch stars undergo major modification during the rapid transition to planetary nebulae (PN). In this proto-PN phase, fast winds emitted by the central star compress and accelerate the neutral envelope, and as the central star evolves to higher temperatures it emits ultraviolet radiation that photo-dissociates the neutral gas and gradually ionizes the expanding envelope. The combined action of the winds and the stellar radiation shapes the circumstellar envelope which later characterizes the morphology during the planetary nebula phase (Huggins et al. 1996).

Send offprint requests to: P. Cox, e-mail: cox@ias.fr

* Based on observations collected at the Canada-FranceHawaii Telescope, operated by the National Research Council of Canada, the Centre National de la Recherche Scientifique de France, and the University of Hawaii.
NGC 7027 is an important archetype in the study of $\mathrm{PN}$ formation because it is relatively young, with strong line and continuum emission from both the ionized and neutral gas. The dense, inner envelope has been ionized by the intense radiation field of the hot central star ( $T_{\text {eff }} \sim 200000 \mathrm{~K}$, e.g., Robberto et al. 1993; Latter et al. 2000), but farther out the envelope is still molecular. In the central regions, extended, high-temperature X-ray emission has been detected (Kastner et al. 2001). The morphology of the ionized gas has been described with a prolate ellipsoidal shell, which is expanding radially away from the central, exciting star (e.g., Masson 1989). The molecular hydrogen emission is found in a striking shell structure with fourfold symmetry which loops around the ionized gas (Graham et al. 1993a; Kastner et al. 1994; Cox et al. 1996) and traces the photo-dissociation region 

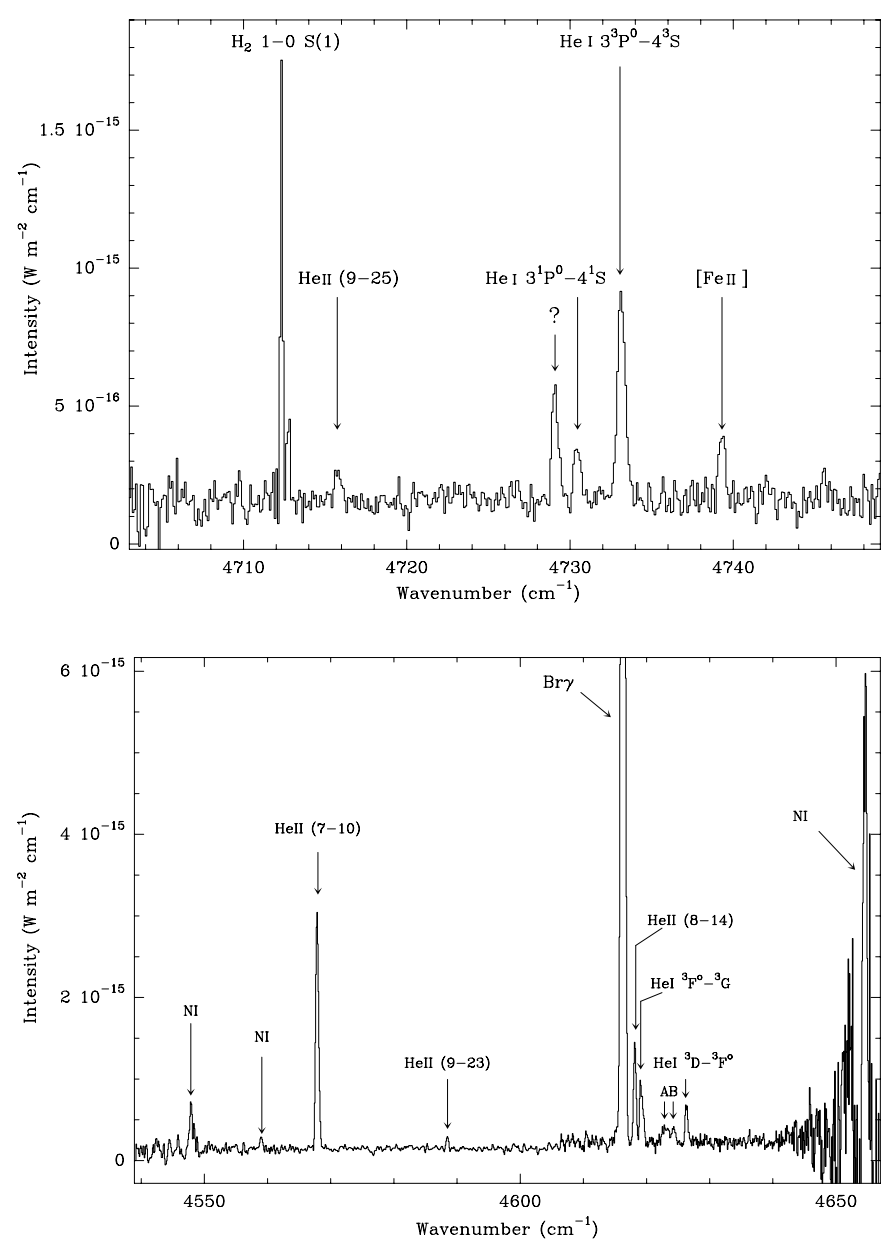

Fig. 1. $B E A R$ spectra at the peak emission of the ionized region in NGC 7027 as measured in the three filters used in this study: the $\mathrm{H}_{2}$ filter from 4708 to $4745 \mathrm{~cm}^{-1}$ (upper panel), the Br $\gamma$ filter from 4585 to $4658 \mathrm{~cm}^{-1}$ and the HeII filter from $4530-4640 \mathrm{~cm}^{-1}$ (lower panel) combined in one spectrum. The emission lines are identified (see Table 1). The lines marked $A$ and $B$ in the lower panel correspond to [FeII $] 3 d^{7} a^{2} \mathrm{H}-3 d^{6}$ $\mathrm{a}^{4} \mathrm{G}$ and HeI $4 \mathrm{f}^{3} \mathrm{~F}^{0}-7 \mathrm{~g}^{3} \mathrm{G}$. The Br $\gamma$ line is off-scale and peaks at $2.32 \times 10^{-14} \mathrm{~W} \mathrm{~m}^{-2} \mathrm{~cm}^{-1}$. Note that the continuum has not been subtracted and that the $\mathrm{H}_{2}$ line (upper panel) shows two velocity components. The lower signal-to-noise ratio in the spectrum around $4650 \mathrm{~cm}^{-1}$ (lower panel) is due to the fact that this region is at the border of the $\mathrm{Br} \gamma$ filter.

(PDR) between the ionization front and the inner border of the expanding molecular envelope.

Recent HST/NICMOS observations have provided further information on the morphology of the molecular hydrogen emission in NGC 7027 (Latter et al. 2000). The emission is found to arise in a very thin layer, and at small scales is structured and filamentary, indicating the presence of inhomogeneities in the PDR gas. The large scale geometry has been interpreted using a biconical model by Latter et al. (2000). The first measurements of the $\mathrm{H}_{2}$ kinematics have also shown that the expansion of the PDR is closely linked to that of both the ionized gas and the outer molecular envelope (Cox et al. 1996; Latter et al. 2000).

The present paper reports high resolution spectroimaging of NGC 7027 that provides for the first time a detailed view of both the morphology and kinematics of the $\mathrm{H}_{2}$ and the ionized gas. In Sect. 2, we discuss the observations and the data. The morphology and kinematics of the ionized and molecular gas are described in Sect. 3. In Sect. 4, we present simple models for the $\mathrm{Br} \gamma$ and $\mathrm{H}_{2}$ emission which constrain the geometry and kinematics of the nebula and the PDR. These models do not account for the observed inhomogeneities nor for important deviations from axi-symmetry which are explained in Sect. 5 in terms of the action of collimated outflows. In Sect. 6, we derive the physical properties of the $\mathrm{H}_{2}$ emitting gas using a PDR model which includes a detailed treatment of the thermal balance and of the chemistry. Conclusions are given in Sect. 7.

\section{Observations}

The observations were made on October 24, 1999 and October 7 and 8, 2000 using the BEAR Imaging FTS at the $\mathrm{f} / 35$ infrared focus of the 3.60-meter Canada-FranceHawaii Telescope. A detailed description of the properties of $B E A R$ is given by Simons et al. (1994) and Maillard (1995) - see Maillard (2000) for an updated review. The instrument $B E A R$ results from the coupling of the CFHT step-scan high resolution facility FTS (Maillard \& Michel 1982) with a $256 \times 256 \mathrm{HgCdTe}$ facility camera. The field of view is circular with a diameter of $24^{\prime \prime}$, and the plate scale on the detector is $0.35 /$ pixel. During the observations, the seeing at $2 \mu \mathrm{m}$ was typically 0.5 . In the camera, narrow-band filters gave access to the following spectral ranges: i) $4705-4745 \mathrm{~cm}^{-1}$ which includes the $\mathrm{H}_{2}$ 1-0 S(1) $4712.9 \mathrm{~cm}^{-1}(2.12 \mu \mathrm{m})$ and the $4733.5 \mathrm{~cm}^{-1}$ He I $4^{3} \mathrm{~S}-3^{3} \mathrm{P}^{0}$ lines (used in Oct. 1999), ii) $4585-4658 \mathrm{~cm}^{-1}$ centered on the $4616.6 \mathrm{~cm}^{-1}(2.16 \mu \mathrm{m})$ $\mathrm{Br} \gamma$ line, and iii) $4530-4640 \mathrm{~cm}^{-1}$ which includes the $4568.1 \mathrm{~cm}^{-1}(2.19 \mu \mathrm{m})$ He II $(7-10)$ line (the last two filters were used in Oct. 2000). For the observations with each filter, the raw data consist of a cube of 1150 planes with an integration time of $9 \mathrm{~s}$ per image, an image being taken at each step of the interferometer. The maximum path difference corresponds to a resolution $(F W H M)$ of $0.137 \mathrm{~cm}^{-1}$, i.e. a velocity resolution of $8.9,9.1$ and $15.6 \mathrm{~km} \mathrm{~s}^{-1}$ for the $\mathrm{H}_{2}, \mathrm{Br} \gamma$ and He II $\left(4568 \mathrm{~cm}^{-1}\right)$ filters, respectively, an improvement of a factor $\sim 6$ compared to the earlier observations of NGC 7027 reported by Cox et al. (1996). Data cubes on the calibration star HD $162208\left(m_{K}=7.11\right)$ from Elias et al. (1982) were obtained at lower resolution for calibration purposes.

The data reduction was carried out with a package called bearprocess originally written by F. Rigaut; the basic operations and modifications made to the initial version are described by Maillard (2000). The reduction 


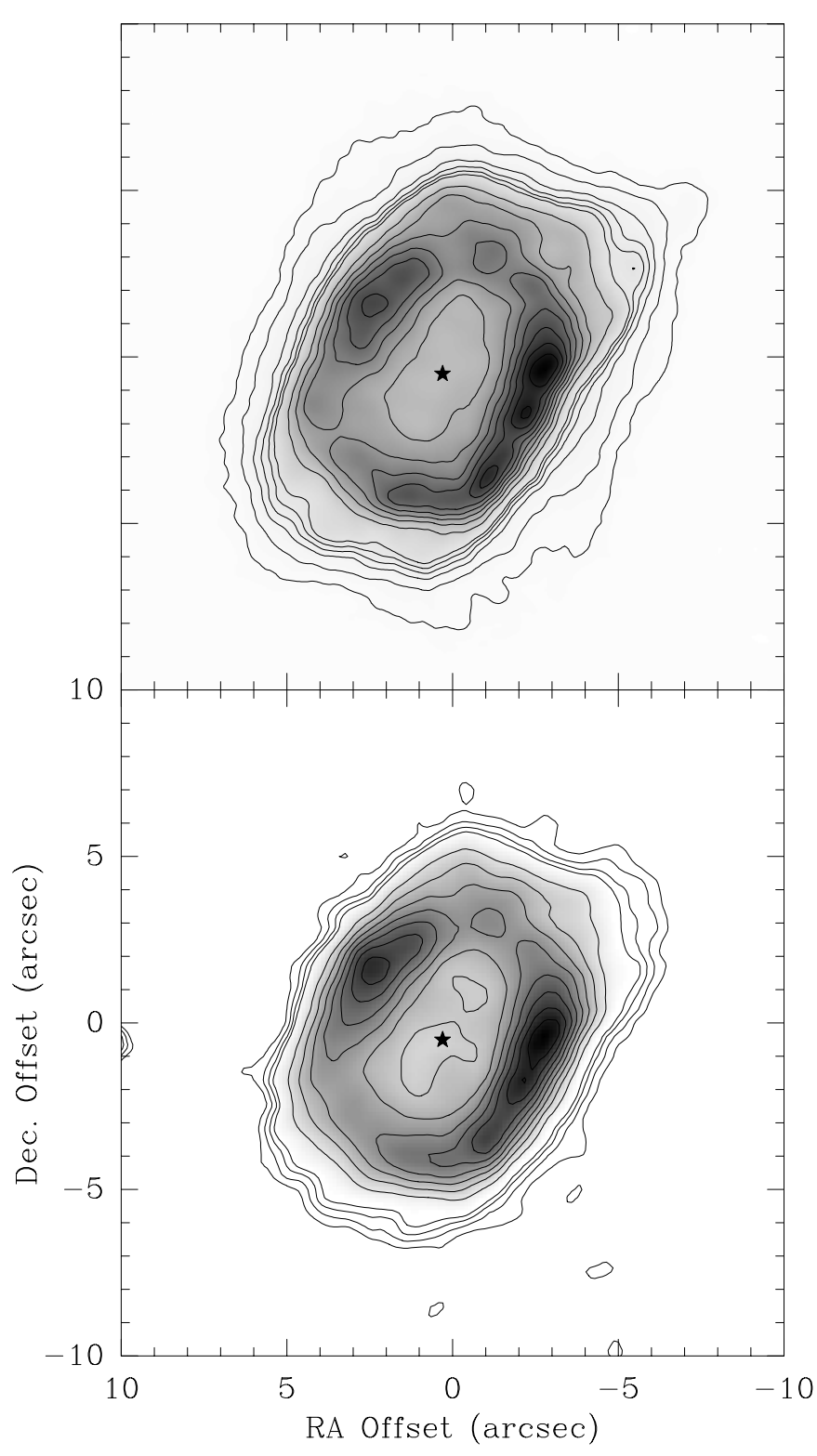

Fig. 2. BEAR images of the ionized cavity in NGC 7027 . Bottom: image in the $2 \mu \mathrm{m}$ continuum. Top: velocity integrated $\operatorname{Br} \gamma$ line at $2.16 \mu \mathrm{m}\left(4616.6 \mathrm{~cm}^{-1}\right)$. The contours are from 4,6 , 8,10 to $100 \%$ by $10 \%$ of the peak intensity of the maps with an additional contours at 1 and $2 \%$ for the $\operatorname{Br} \gamma$ map to show the halo emission of the ionized gas. The peak intensities of the $\operatorname{Br} \gamma$ and $2 \mu \mathrm{m}$ continuum emission are $4.12 \times 10^{-5}$ and $4.63 \times 10^{-6} \mathrm{Wm}^{-1} \mathrm{sr}^{-1}$, respectively. The offsets are given with respect to the central star (shown as a filled symbol) whose coordinates are: 21:07:01.5 42:14:09.9 (J2000).

includes the usual procedures ${ }^{1}$ of flat-fielding the images, sky subtraction, correction for bad pixels, and registration of each frame to the first one to correct for turbulent motions and flexure drifts. In order to take full advantage of the quality of the seeing and to accurately register the images, the raw frames were spatially oversampled by a

\footnotetext{
${ }^{1}$ In the case of the $\mathrm{H}_{2}$ data cube, a special processing was applied to filter out correlated noise which was due to a grounding problem in the camera which occurred during the observations.
}

factor of 4 (from $70 \times 70$ to $142 \times 142$ pixels). All the interferograms were extracted from this corrected cube and the corresponding spectra were computed by FFT, yielding in the present case a 1260-plane cube. From the division of the spectral cube by the reference spectrum, the final cube was produced, corrected for the instrumental and atmospheric transmission. In order to fully reconstruct the line profiles and to apply the instrumental phase correction through the field, two special, oversampled cubes were computed with 882 and 841 frames over small domains around the $\mathrm{H}_{2}$ line $\left(\simeq 12.5 \mathrm{~cm}^{-1}\right)$ and the $\operatorname{Br} \gamma$ line $\left(\simeq 9.5 \mathrm{~cm}^{-1}\right)$. In these cubes, the separation between frames corresponds to a velocity resolution of $\sim 0.90 \mathrm{~km} \mathrm{~s}^{-1}$, allowing us to estimate the line positions to better than $0.5 \mathrm{~km} \mathrm{~s}^{-1}$.

Figure 1 shows sample $B E A R$ spectra from the $\mathrm{H}_{2}, \mathrm{Br} \gamma$ and He II data cubes, towards a position of strong emission from the ionized gas at offsets $\left(-3^{\prime \prime}, 0^{\prime \prime}\right)$ from the central star (see Fig. 2). Besides the strong lines of $\mathrm{H}_{2}, \mathrm{Br} \gamma$, He I and HeII, the spectra include the continuum and a series of weaker emission lines which originate in the ionized gas (see Sect. 3.3). Note that all lines are resolved. The full $\mathrm{H}_{2}, \mathrm{Br} \gamma$, and He II cubes consist of $\sim 3600$ spectra with similar noise levels, which allow us to derive the distribution and the kinematics of each of the emission lines together with the morphology of the continuum emission. The absolute pointing of the data cubes was determined by matching the observed continuum and the $\operatorname{Br} \gamma$ images with the high resolution radio continuum map from Hajian et al. (1993).

\section{Results}

\subsection{The ionized gas}

The distribution of the ionized gas in NGC 7027 is shown in Fig. 2 in images of the $\operatorname{Br} \gamma$ and continuum emission produced from the $B E A R$ data. The $\operatorname{Br} \gamma$ image is the velocity integrated emission in the line with the continuum subtracted, and the $2 \mu \mathrm{m}$ continuum image has been obtained by integrating the full bandpass of the $\operatorname{Br} \gamma$ filter after removing all the emission lines and thus shows only the near-infrared continuum. Because of the high spectral resolution of the $B E A R$ data, these images are essentially free of all contamination.

Both the $\operatorname{Br} \gamma$ emission and the continuum are distributed in an approximately elliptical ring with brightening on the minor axis, which is commonly seen in optical images of PNe. The dimensions are $\sim 6^{\prime \prime} \times 12^{\prime \prime}$ with the major axis oriented at $\mathrm{PA}_{\text {ionized }} \sim 32^{\circ}$. Although the nebula can be described as elliptical to a first approximation, close examination reveals that there are significant deviations. In particular, the sides of the shell are nearly parallel and the ends are distinctly flattened. The brightness along the rim of the shell is also irregular and clumpy: along the western rim there are three peaks, the strongest peak lying about $4^{\prime \prime}$ to the north-west of the central star, and along the east rim, one emission peak is present also to the north with respect to the central 
star. In addition, extended low level emission is present in a roughly rectangular shape with weak extensions to the north-west and south-east (Fig. 2). The size of the $\mathrm{Br} \gamma$ halo is about twice that of the bright shell emission and its orientation is slightly tilted w.r.t. the main axis of the ionized gas $\left(\mathrm{PA}_{\text {halo }} \sim 28^{\circ}\right)$.

The $2 \mu \mathrm{m}$ continuum image shows a morphology which is very similar to that seen in the $\operatorname{Br} \gamma$ line, including the inhomogeneities, the peaks, and the halo emission. This is consistent with the continuum emission arising from thermal emission from the hot ionized gas. The morphology in the $\mathrm{Br} \gamma$ line and near-infrared continuum from the $B E A R$ data is similar to that seen in the radio continuum (e.g., Masson 1989; Roelfsema et al. 1991; Hajian et al. 1993; Bryce et al. 1997) and matches previous nearinfrared mapping results (Woodward et al. 1992; Graham et al. 1993a,b; Cox et al. 1996; Latter et al. 2000).

The kinematics of the ionized gas are shown in the form of channel maps of the $\operatorname{Br} \gamma$ emission (Fig. 3) and velocity-strip maps along the major and minor axes (right panels of Fig. 4). In the channel map at the systemic velocity $v_{\text {sys }} \sim 25 \mathrm{~km} \mathrm{~s}^{-1}$, which corresponds to a cross section in the plane of the sky and is not confused by integration along the line of sight or by model assumptions, the emission of the ionized gas traces a complete, roughly elliptical shell with the exciting star at the center. At redshifted velocities $\left(v>25 \mathrm{~km} \mathrm{~s}^{-1}\right)$, the $\operatorname{Br} \gamma$ emission is strongest along the northern rim of the shell, and as the velocity increases this rim shifts progressively closer to the star. At blue-shifted velocities $\left(v<25 \mathrm{~km} \mathrm{~s}^{-1}\right)$, the reverse is seen, with the $\operatorname{Br} \gamma$ emission distributed along the southern rim of the shell and shifting closer to the star at higher velocities. Around the terminal velocities ( $\sim 0$ and $\sim 50 \mathrm{kms}^{-1}$ ), the overall emission of the ionized gas is still distributed in a shell, but offset from the central star, to the south at red-shifted velocities, and to the north at blue-shifted velocities. The inhomogeneities in the brightness distribution are seen in all the velocity channels. The position-velocity diagrams of the Br $\gamma$ emission further illustrate the velocity gradients already seen in the channel maps and show that the north-west peak is shifted towards more blue-shifted velocities than the south-east peak (upper right panel of Fig. 4), a behaviour consistent with the north-west tilted towards the observer and the south-east tilted away from the observer. The expansion velocity of the ionized gas along the line of sight is $19.5 \pm 0.1 \mathrm{~km} \mathrm{~s}^{-1}$ as estimated by the separation of the double-peaked $\operatorname{Br} \gamma$ spectrum measured towards the centre of the ionized cavity. From this spectrum, the $\mathrm{Br} \gamma$ line broadening is measured to be $27.3 \pm 0.3 \mathrm{~km} \mathrm{~s}^{-1}$ (FWHM), after correction for the instrumental line shape. The channel and velocity-strip maps in the $\mathrm{Br} \gamma$ emission line are in agreement with the results of Roelfsema et al. (1991) who presented channel maps and velocity diagrams in the H76 $\alpha$ recombination line.

The extended low level emission is traced in $\operatorname{Br} \gamma$ from 0 to $50 \mathrm{~km} \mathrm{~s}^{-1}$ (Fig. 3). Its size, which is maximum at the systemic velocity (with a nearly rectangular shape), diminishes progressively towards the blue- and red-shifted velocity channels. At velocities around 5 and $45 \mathrm{kms}^{-1}$, the low level emission extends toward the north-west and south-east, respectively. In addition, the $\operatorname{Br} \gamma$ emission traces high-velocity ionized gas which is seen for the first time. The high-velocity gas, which extends $\sim 30 \mathrm{~km} \mathrm{~s}^{-1}$ beyond the terminal velocity, is detected in the north-west (blue-shifted) and south-east (red-shifted) parts of the ionized cavity. The highest velocity gas is clumpy with emission knots located symetrically with respect to the central star (channel maps at -30 and $80 \mathrm{~km} \mathrm{~s}^{-1}$ ).

\subsection{The $\mathrm{H}_{2}$ emission}

The overall distribution of the $\mathrm{H}_{2}$ emission observed with $B E A R$ is shown in the velocity integrated image in Fig. 5. Note that this image contains no emission either from continuum or from nearby emission lines from the ionized gas. The general aspect of the $\mathrm{H}_{2}$ distribution appears limb brightened with emission seen in the equatorial regions. The detailed stucture is complex with multiple lobes and weak $\mathrm{H}_{2}$ emission linking the polar caps along the major axis. The bi-conical shell has dimensions $\sim 10^{\prime \prime} \times 13^{\prime \prime}$ and the position angle of the major axis is $\mathrm{PA}_{\mathrm{H}_{2}} \sim 28^{\circ}$. The four-lobed clover pattern of the $\mathrm{H}_{2}$ emission, which was described in previous studies (Graham et al. 1993b; Kastner et al. 1994; Cox et al. 1996; Latter et al. 2000), shows holes in the brightness distribution, noticeably to the north-west and south-east where weak $\mathrm{H}_{2}$ emission extends beyond the main limb-brightened structure. Despite the complexity, there is a remarkable symmetry in the distribution with respect to the central star. The image shows a striking similarity to the HST image published by Latter et al. (2000). Most of the details present in the HST image are seen in the $B E A R$ data, in particular the intricate series of wisps and filaments around the main structure as well as the outer north and south loops.

The kinematics of the $\mathrm{H}_{2}$ emission is shown in the form of position-velocity diagrams along the major and minor axes (left panels of Fig. 4) and velocity channel maps in Fig. 6. The $\mathrm{H}_{2}$ line width is seen to be quite narrow in Fig. 4 and we estimate it by extracting a spectrum on a small aperture along the line of sight through the center. The line profile shows two components, each with a width of $\sim 9.5 \mathrm{~km} \mathrm{~s}^{-1}(F W H M)$, much narrower than that seen in the ionized gas.

In Fig. 6, the channel map of the $\mathrm{H}_{2}$ emission at the systemic velocity $\left(v_{\text {sys }} \sim 25 \mathrm{~km} \mathrm{~s}^{-1}\right)$ shows a completely hollow shell, whose rim traces the four lobed pattern seen in the velocity integrated image. The emission is brightest at the equator or waist, where it shows distinct indentations towards the central star. The brightness distribution is inhomogeneous and weaker emission is seen protruding out from the north-west and south-east edges.

At red-shifted velocities $\left(v>25 \mathrm{~km} \mathrm{~s}^{-1}\right)$, the $\mathrm{H}_{2}$ emission shifts towards the south. The southern rim is traced out to the highest velocities, but the brightness of the 


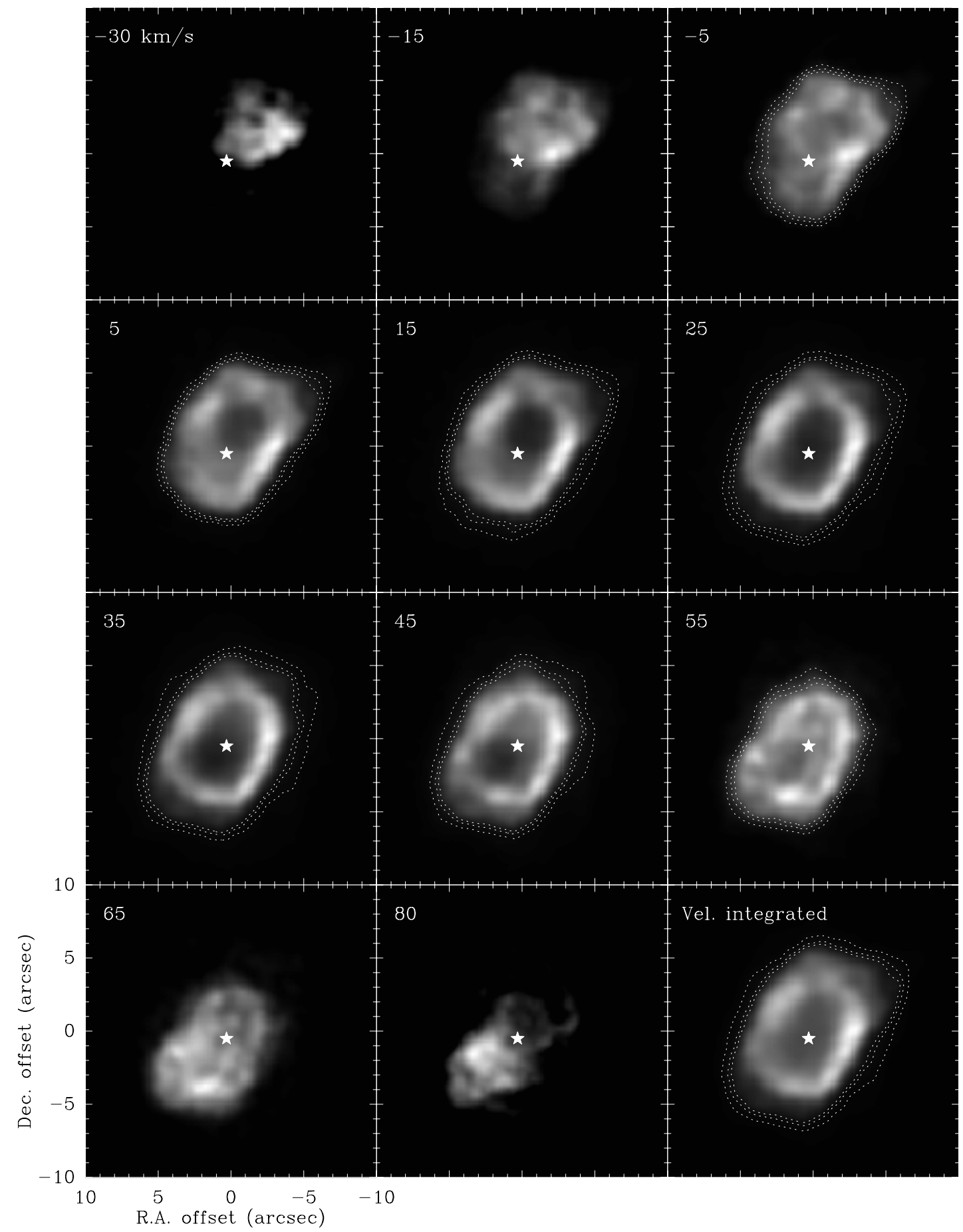

Fig. 3. Velocity channel maps of emission in the $\operatorname{Br} \gamma$ line at $4616.6 \mathrm{~cm}^{-1}(2.16 \mu \mathrm{m})$ in NGC 7027 . The central velocity $\left(v_{\mathrm{LSR}}\right)$ of each channel is given in the upper left of each panel and the systemic velocity is at $\sim 25 \mathrm{~km} \mathrm{~s}^{-1}$. The channel widths are $10 \mathrm{~km} \mathrm{~s}^{-1}$, except for the extreme velocity channels (centered at -30 and $80 \mathrm{~km} \mathrm{~s}^{-1}$ ) where the width is $20 \mathrm{~km} \mathrm{~s}$. The offsets are given with respect to the central star (shown as a filled star symbol). The right panel at the bottom shows the velocity integrated Br $\gamma$ emission - see Fig. 2. The dashed contours show the extended low level Br $\gamma$ emission $(2,4$, and $6 \%$ of the peak intensity of the $\operatorname{Br} \gamma$ emission at the systemic velocity for each panel and the velocity integrated $\operatorname{Br} \gamma$ emission in the lower right panel).

northern rim diminishes progressively and is only weakly detected at velocities around $40 \mathrm{~km} \mathrm{~s}^{-1}$. At intermediate red-shifted velocities, the equator fills in just to the north of the central star; its brightness becomes strongest around $40 \mathrm{~km} \mathrm{~s}^{-1}$ and diminishes again at higher veloc- ities. The hole in the south-east edge widens up as the velocity increases. Between 35 and $45 \mathrm{~km} \mathrm{~s}^{-1}$, the eastern rim becomes weaker and emission fills in the south-west part of the nebula. At the highest velocities, weak $\mathrm{H}_{2}$ emission extends from the position of the central star towards 
Table 1. Atomic lines observed in NGC 7027 with BEAR.

\begin{tabular}{|c|c|c|c|c|c|}
\hline \multicolumn{2}{|c|}{$\left(\mathrm{cm}^{-1}\right)$} & & Transition & $\begin{array}{l}\text { Line Flux }{ }^{\dagger} \\
\left(10^{-12} \mathrm{~W} \mathrm{~m}^{-2}\right)\end{array}$ & Ref. \\
\hline \multicolumn{6}{|c|}{ HeII data cube } \\
\hline 4548.33 & 4547.9 & $\mathrm{NI}^{\ddagger}$ & $2 \mathrm{p}^{2}\left({ }^{3} \mathrm{P}_{2}\right) 4 \mathrm{f}-2 \mathrm{p}^{2}\left({ }^{3} \mathrm{P}\right) 7 \mathrm{~d}^{4} \mathrm{P}$ & 0.37 & $\begin{array}{l}\mathrm{a}, \mathrm{b} \\
\mathrm{c}, \mathrm{d}\end{array}$ \\
\hline 4559.34 & 4558.9 & $\mathrm{~N}_{\mathrm{I}}$ & $2 \mathrm{p}^{2}\left({ }^{3} \mathrm{P}_{1}\right) 4 \mathrm{f}-2 \mathrm{p}^{2}\left({ }^{3} \mathrm{P}\right) 7 \mathrm{~d}^{4} \mathrm{~F}$ & 0.07 & \\
\hline 4568.13 & 4568.06 & He II & $7-10$ & 1.67 & $\mathrm{~b}$ \\
\hline 4582.82 & 4582.82 & He I & $4 \mathrm{~d}^{3} \mathrm{D}-7 \mathrm{p}^{3} \mathrm{P}^{0}$ mult & 0.06 & \\
\hline 4588.85 & 4588.75 & He II & $9-23$ & 0.06 & \\
\hline \multicolumn{6}{|c|}{$\mathrm{Br} \gamma$ data cube } \\
\hline 4616.59 & 4616.55 & $\mathrm{Br} \gamma$ & & 16.03 & \\
\hline 4618.50 & 4618.44 & He II & $8-14$ & 0.62 & \\
\hline 4619.52 & 4619.49 & He I & $4 \mathrm{f}^{3} \mathrm{~F}^{0}-7 \mathrm{~g}^{3} \mathrm{G}$ mult & 0.51 & $\mathrm{a}$ \\
\hline 4623.10 & 4623.01 & {$[\mathrm{Fe} \mathrm{II}]$} & $3 d^{7} a^{2} H-3 d^{6} a^{4} G$ & 0.19 & \\
\hline 4624.74 & 4624.72 & $\mathrm{He} \mathrm{I}$ & $4 \mathrm{~d}^{1} \mathrm{D}-7 \mathrm{f}^{1} \mathrm{~F}^{0}$ & 0.15 & $\mathrm{a}$ \\
\hline 4626.75 & 4626.69 & He I & $4 \mathrm{~d}^{3} \mathrm{D}-7 \mathrm{f}^{3} \mathrm{~F}^{0}$ mult & 0.31 & $\mathrm{a}$ \\
\hline 4654.95 & 4654.45 & $\mathrm{~N}_{\mathrm{I}}$ & $2 \mathrm{p}^{2}\left({ }^{3} \mathrm{P}_{0}\right) 4 \mathrm{f}-2 \mathrm{p}^{2}\left({ }^{3} \mathrm{P}\right) 7 \mathrm{~d} 4 \mathrm{~F}$ & 4.61 & \\
\hline \multicolumn{6}{|c|}{$\mathrm{H}_{2}$ data cube } \\
\hline 4716.19 & 4716.18 & He II & $9-25$ & 0.05 & \\
\hline 4729.47 & 4729.5 & O I? & $4 d^{5} D^{0}-7 f^{5} F$ & 0.19 & \\
\hline 4730.80 & 4730.86 & He I & $3 \mathrm{p}^{1} \mathrm{P}^{0}-4 \mathrm{~s}^{1} \mathrm{~S}$ & 0.06 & $\mathrm{a}$ \\
\hline 4733.52 & 4733.53 & He I & $3 \mathrm{p}^{3} \mathrm{P}^{0}-4 \mathrm{~s}^{3} \mathrm{~S}$ & 0.46 & $\mathrm{a}, \mathrm{b}$ \\
\hline 4739.72 & 4739.82 & {$[\mathrm{Fe} \mathrm{II}]$} & $3 d^{6} a^{4} H-3 d^{6} b^{2} H$ & 0.09 & \\
\hline
\end{tabular}

Notes. The identifications of the lines are based on the atomic data base maintained by Peter van Hoof at the following Web page: http://www.pa.uky.edu/ $\sim$ peter/atomic/index.html

(a) Observed in Hb 12 by Luhman \& Rieke (1996). (b) Observed in NGC 7027 by Smith et al. (1981). (c) Observed in NGC 7027 by Geballe et al. (1991). (d) Mapped in NGC 7027 by Cox et al. (1996).

$\dagger$ The atomic line fluxes have been measured in a region of $8^{\prime \prime} 1 \times 8^{\prime \prime} .1$ containing most of the ionized cavity. Errors on the line intensities are estimated to be a few percent.

${ }^{\ddagger}$ Identified as $[\mathrm{K} \mathrm{III}]{ }^{3} \mathrm{P}_{1}-{ }^{3} \mathrm{P}_{2}$ by Dinerstein (2001).

the south. At blue-shifted velocities all these features are seen, but with North and South transposed. The positionvelocity diagram (Fig. 4) shows an elongated shape along the major axis with a clear waist at the equator and openings in the north-west and south-east which are symmetric with respect to the central star, in agreement with the results of Latter et al. (2000); along the minor axis the velocity-strip has a closed elongated structure. As in the case of the ionized gas, the north-west $\mathrm{H}_{2}$ emission is more blue-shifted than the emission in the south-east indicating that the northern part of the nebula is tilted with respect to the line of sight towards the observer.

\subsection{Atomic lines}

In addition to the $\mathrm{H}_{2} 1-0 \mathrm{~S}(1)$ and $\mathrm{Br} \gamma$ lines, the three $B E A R$ data cubes contain 16 additional lines, including strong emission in He I $3 \mathrm{p}^{3} \mathrm{P}^{0}-4 \mathrm{~s}^{3} \mathrm{~S}$ and He II $(7-10)$ (Fig. 1 and Table 1). The BEAR data allows us to determine the distribution and kinematics for each of these lines, and they are found to be similar to those of $\operatorname{Br} \gamma$. This demonstrates that the lines originate in the ionized cavity of NGC 7027, and implies that the carriers are atomic species. The wavenumbers of the lines after correction for the Doppler shift (of the Earth's orbital motion and the systemic velocity of NGC 7027) are listed in Table 1 together with their identifications.

The helium recombination lines (He I and He II) are prominent in the BEAR spectra of NGC 7027 with 6 He I and $4 \mathrm{He}$ II lines detected. The strongest of these lines have previously been observed in NGC 7027 (Smith et al. 1981) or in the young planetary nebula $\mathrm{Hb} 12$ (Luhman \& Rieke 1996) - see Table 1 - but to our knowledge, none of the weaker helium recombination lines listed in Table 1 have been reported in any astronomical source. The many lines allows us to test the relative line strengths against Helium recombination theory (case B). All ratios $(\mathrm{HeI} / \mathrm{Br} \gamma$ and $\mathrm{HeII} / \mathrm{Br} \gamma)$ are consistent with $T_{\mathrm{e}} \sim$ $1.5-2.0 \times 10^{4} \mathrm{~K}$ and an average density $n_{\mathrm{e}} \sim 5 \times 10^{4} \mathrm{~cm}^{-3}$, in good agreement with previous estimates based on radio 
$\mathrm{H}_{2}$

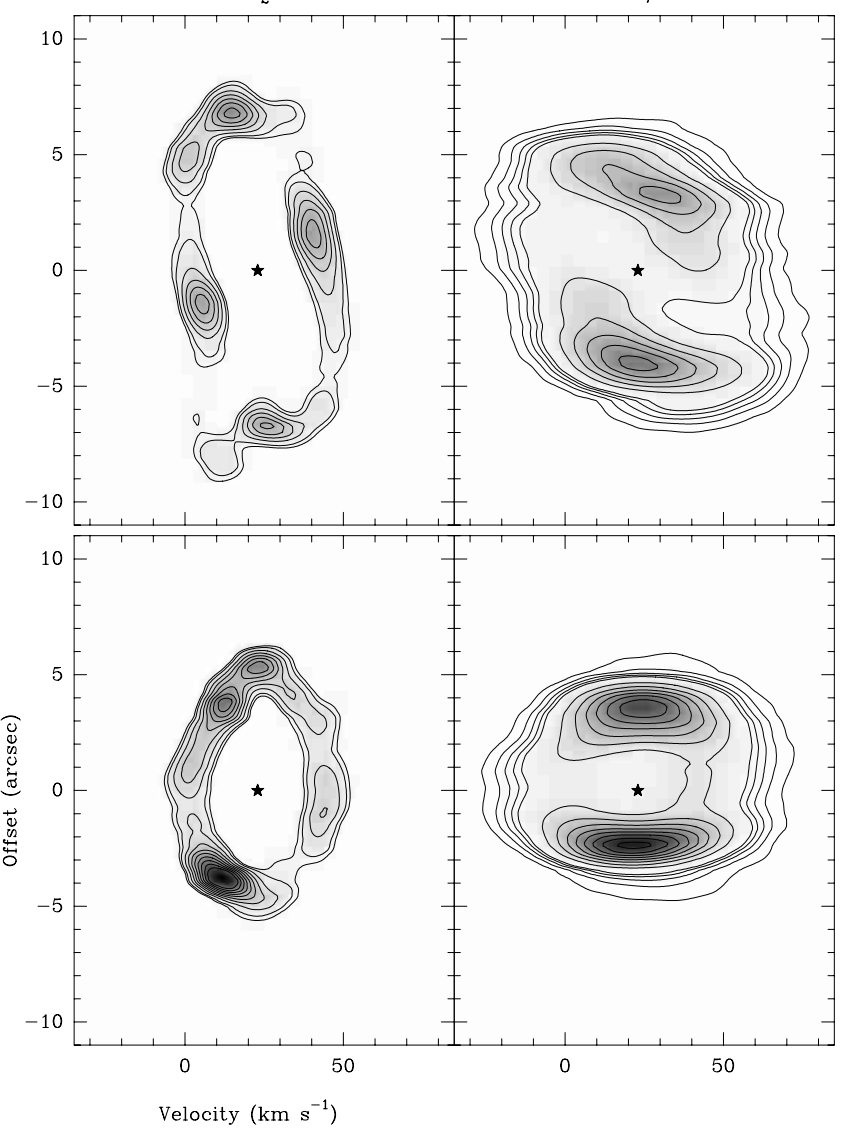

Fig. 4. $\mathrm{H}_{2}$ 1-0 S(1) and $\mathrm{Br} \gamma$ velocity-strip maps of NGC 7027 along the major (upper panels) and minor axis (lower panels). The offsets are given with respect to the central star's position (denoted by a filled star symbol).

(Masson 1989; Roelfsema et al. 1991) and optical observations (e.g., Péquignot \& Baluteau 1994) for which He II $4686 \AA / \mathrm{H} \beta \approx 0.4$ (Kaler et al. 1976). The sizes of the $\mathrm{H}$ and $\mathrm{He}$ recombination zones (both $\mathrm{He}^{+}$and $\left.\mathrm{He}^{++}\right)$are about the same, as expected for a central star of $T_{\text {eff }} \sim 200000 \mathrm{~K}$, i.e. hot enough to produce $\mathrm{He}^{+}$and $\mathrm{He}^{++}$throughout the HiI region.

The two [Fe II] lines are not unexpected in NGC 7027. The size of the $\mathrm{Fe}^{++}$zone is coincident with the $\mathrm{H}$ and He recombination regions and its morphology is similar to that of $\mathrm{Br} \gamma$, showing the same distribution of knots and peaks.

Three lines are identified as N I including the line observed at $4548.33 \mathrm{~cm}^{-1}(2.198 \mu \mathrm{m})$ which was reported previously in NGC 7027 (Smith et al. 1981; Geballe et al. 1991; Cox et al. 1996). The identification of these lines is based on the close correspondence of the observed positions with the $\mathrm{N}$ I lines. The $4654.4 \mathrm{~cm}^{-1} \mathrm{~N}$ I $2 \mathrm{p}^{2}\left({ }^{3} \mathrm{P}_{0}\right) 4 \mathrm{f}-$ $2 \mathrm{p}^{2}\left({ }^{3} \mathrm{P}\right) 7 \mathrm{~d} 4 \mathrm{~F}$ line is remarkably strong, with a line flux $\approx 1 / 3$ of the $\operatorname{Br} \gamma$ line (see Fig. 1). It would be interesting to search for other near-infrared N I transitions in NGC 7027 to confirm the present identification. We note that the emission line at $4548.33 \mathrm{~cm}^{-1}$ remained unidentified, although the studies by Geballe et al. (1991) and

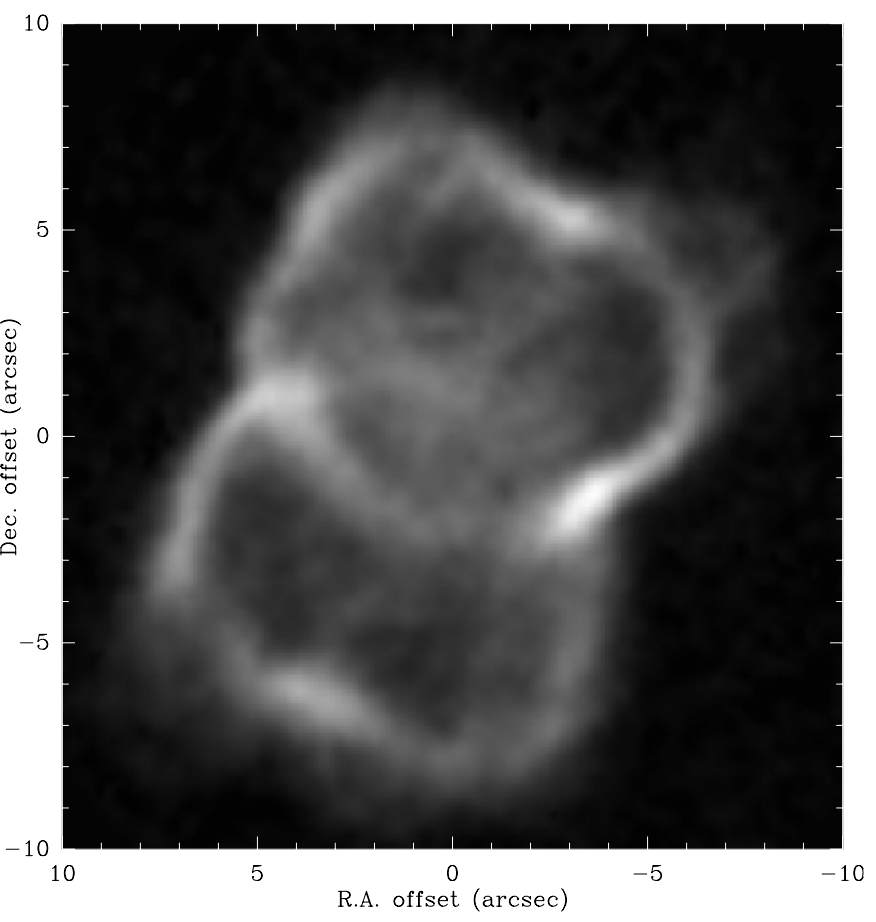

Fig. 5. Image of NGC 7027 in the velocity integrated $\mathrm{H}_{2}$ $1-0 \mathrm{~S}(1)$ transition. The offset are given with respect to the central star. The peak intensity is $2.3 \times 10^{-6} \mathrm{~W} \mathrm{~m}^{-2} \mathrm{sr}^{-1}$.

Cox et al. (1996) provided evidence that it is emitted in the ionized cavity of NGC 7027. The line has also been reported in $\mathrm{Hb} 12$ and in the Orion Nebula (Luhman \& Rieke 1996). Recently, Dinerstein (2001) identified this line with the fine-structure ${ }^{3} \mathrm{P}_{1}-{ }^{3} \mathrm{P}_{2}$ transition of $\mathrm{Kr}^{+2}$, an identification corroborated by the detection of the ${ }^{1} \mathrm{D}_{2}-{ }^{3} \mathrm{P}_{2} 6828 \AA$ emission line in the optical spectra of NGC 7027 and IC 5117, another planetary nebula displaying the $4548.33 \mathrm{~cm}^{-1}$ line. The observed flux of the $4548.33 \mathrm{~cm}^{-1}$ line relative to the $\operatorname{Br} \gamma$ line in IC 5117 is 0.030, comparable to the ratio found in NGC 7027 (0.023). Further observational and theoretical studies would be helpful to see if the $4548.33 \mathrm{~cm}^{-1}$ line could be a blend of emission lines from $\mathrm{Kr}^{+2}$ and N I.

The relatively strong line observed at $4729.47 \mathrm{~cm}^{-1}$ is tentatively identified with the $4 \mathrm{~d}^{5} \mathrm{D}^{0}-7 \mathrm{f}^{5} \mathrm{~F}$ transition of $\mathrm{O}$. It is the first time that this line is reported in an astronomical source. Its identification is based on the strict coincidence of the observed position with the strong O I line.

The richness of the near-infrared spectrum of NGC 7027 is striking. High spectral resolution studies of this planetary nebula at infrared wavelengths would be desirable to further explore its atomic emission spectrum, similarly to what has been done in the visible (Péquignot \& Baluteau 1994). Such studies would provide a useful data set to test against available atomic data of poorly studied species and/or transitions. 


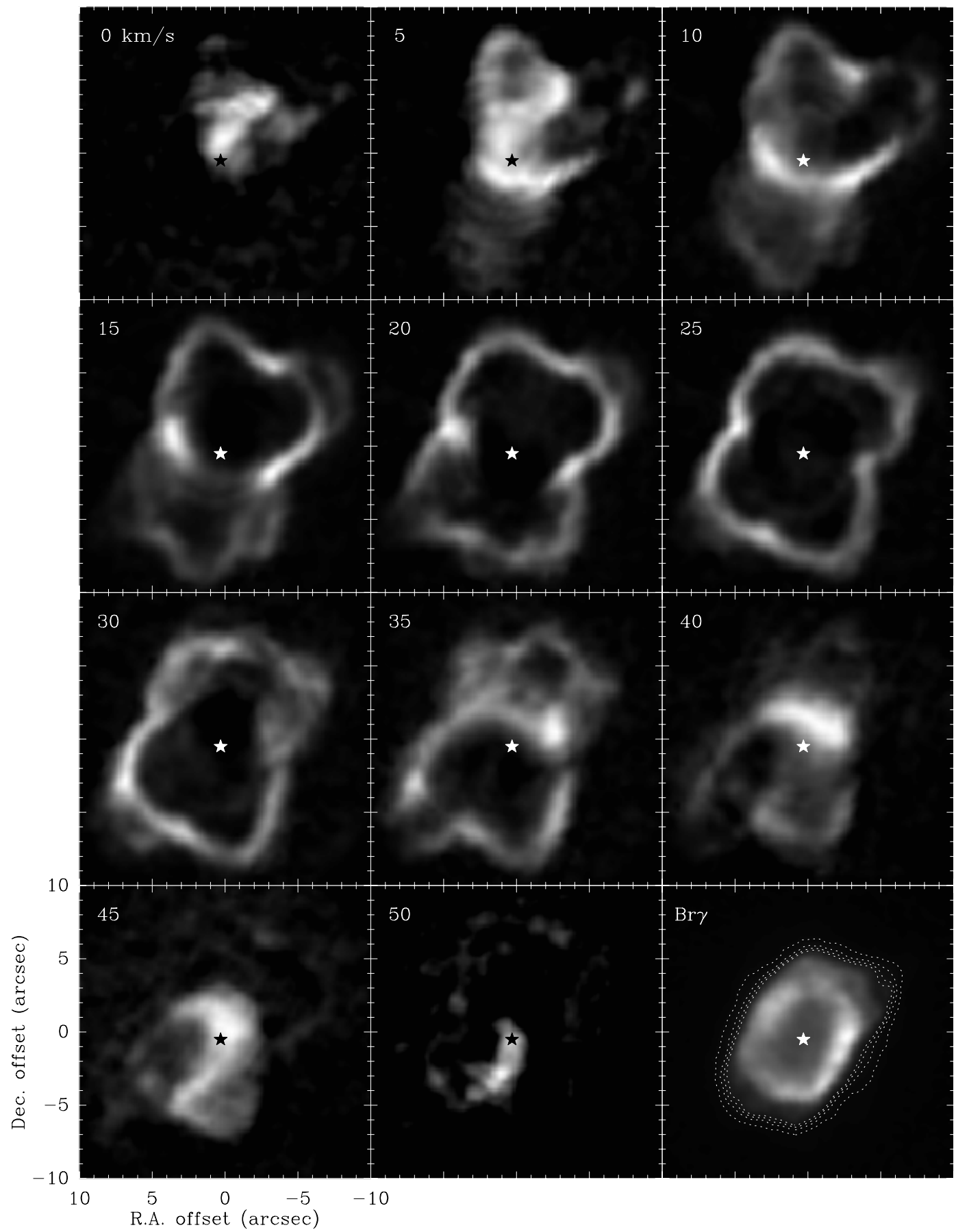

Fig. 6. Velocity channels maps of emission in the $1-0 \mathrm{~S}(1) \mathrm{H}_{2}$ line at $4712.9 \mathrm{~cm}^{-1}(2.12 \mu \mathrm{m})$ in NGC 7027 . The central velocity $\left(v_{\mathrm{LSR}}\right)$ of each channel is given in the upper left of each panel. The channel widths are $5 \mathrm{~km} \mathrm{~s}^{-1}$. The offsets are given with respect to the central star (shown as a filled star symbol). The bottom, right panel shows the velocity integrated Br $\gamma$ emission (from Fig. 2) for comparison.

\section{Morphology and kinematics of NGC 7027}

The observations of NGC 7027 described above show striking differences in morphology between the ionized gas and the $\mathrm{H}_{2}$ emission, which have been described in previous studies of the nebula (Graham et al. 1993a,b; Kastner et al. 1994; Cox et al. 1996; Latter et al. 2000). The present data provide detailed kinematics for each of these components, and reveal new features that underscore the complex early development of PNe.

\subsection{Overview of the nebula}

An overview of the relation between the ionized gas in NGC 7027 and the surrounding neutral gas is shown in Fig. 7. In the lower panel, the integrated intensity image in $\mathrm{Br} \gamma$ is superposed on that of $\mathrm{H}_{2}$. The ionized gas is 

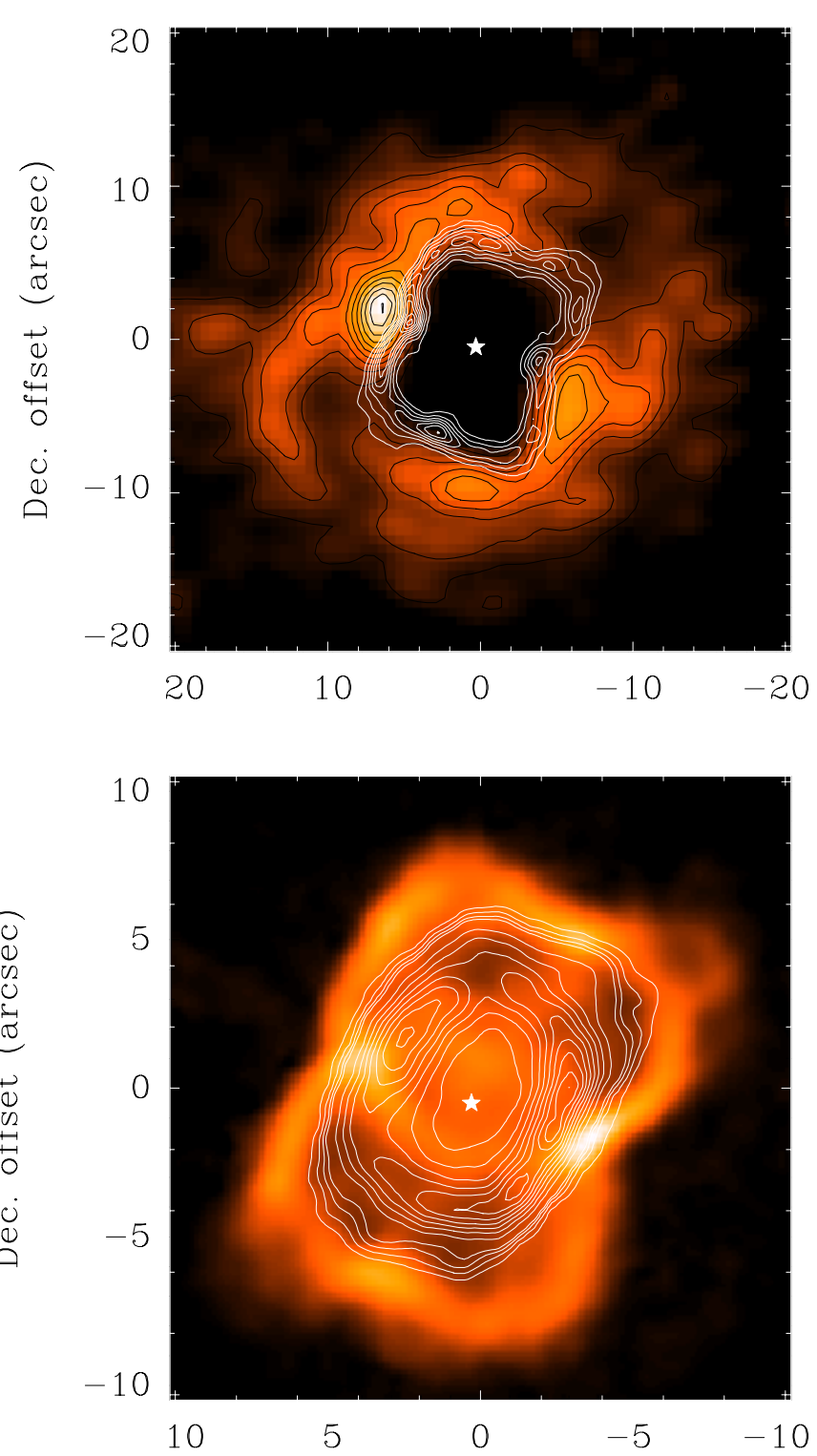

\section{R.A. offset (arcsec)}

Fig. 7. Upper panel: comparison of the channel map at the systemic velocity $\left(25 \mathrm{~km} \mathrm{~s}^{-1}\right)$ in the $\mathrm{CO}(1-0)$ line (from Graham et al. 1993b) (grey scale) with that in the 1-0 S(1) $\mathrm{H}_{2}$ line at $4712.9 \mathrm{~cm}^{-1}$ (contours). The channel widths are $5 \mathrm{~km} \mathrm{~s}^{-1}$. Lower panel: comparison of the velocity integrated $\mathrm{H}_{2}$ line emission with the $\operatorname{Br} \gamma$ emission (contours). The offsets are given with respect to the central star (shown as a filled star symbol).

centered within the lobed $\mathrm{H}_{2}$ structure with the bright minor axis in $\mathrm{Br} \gamma$ corresponding to the $\mathrm{H}_{2}$ waist. At low intensities, the ionized gas fills the $\mathrm{H}_{2}$ cavity to the southeast and north-west. The axis of the $\mathrm{H}_{2}$ emission lies at $\mathrm{PA} \sim 28^{\circ}$ and that of the ionized gas is slightly larger $\mathrm{PA} \sim 32^{\circ}$. Both components are seen to form thin layers $\left(\sim 0 ., 5-0^{\prime \prime} .6\right.$ or $\Delta R \sim(6-7) \times 10^{15} \mathrm{~cm}$ at the adopted distance of 880 pc - see, e.g., Hajian et al. 1993). The thin shell structure supports the interpretation that much of the variation in the continuum and velocity-integrated images is due to limb brightening, where the shell surfaces are seen at different orientations along different lines of sight.

The $\mathrm{H}_{2}$ shell is not an isolated component, but forms part of an extended circumstellar envelope that has been observed out to $70^{\prime \prime}$ from the central star in CO (e.g., Bieging et al. 1991). The relation of the $\mathrm{H}_{2}$ emission to the extended envelope is shown in the upper panel of Fig. 7, where the $\mathrm{H}_{2}$ velocity channel map at the systemic velocity is compared to that of the $\mathrm{CO}(1-0)$ emission observed by Graham et al. (1993b). It can be seen that the $\mathrm{H}_{2}$ forms the inner boundary of the extended envelope, consistent with expectations for the excitation of $\mathrm{H}_{2}$ in a thin PDR, as discussed in Sect. 6.

The channel maps (Figs. 3 and 6) and the positionvelocity strip maps along the major axis (Fig. 4) indicate that both the ionized and neutral parts of the nebula are tilted in the same sense with respect to the line of sight: with the north-west tilted towards the observer and the south-east tilted away from the observer. This general orientation is consistent with previous studies of the ionized gas (e.g., Roelfsema et al. 1991) and the $\mathrm{H}_{2}$ emission (Latter et al. 2000). We note that a previous conclusion by Cox et al. (1996) that the northern polar axis is tilted away from the observer is wrong. It was based on low resolution $\mathrm{H}_{2}$ data obtained with $B E A R$, which is superseded by the observations reported here.

\subsection{Kinematic models}

In order to help interpret the observations and to study the kinematic structure of the nebula, we have constructed some simple geometrical models for the ionized and $\mathrm{H}_{2}$ emitting gas. The models are assumed to be axisymmetric, with a radial velocity field. For a given set of model parameters, we calculate velocity integrated images, channel maps, and position-velocity diagrams, and from comparison with the observations it is possible to determine useful constraints on the properties of the nebula. The models are idealized, and do not take into account the observed inhomogeneities, nor important deviations from axi-symmetry described in Sect. 5 .

\subsubsection{The ionized gas}

A standard model for the ionized gas of PNe with elliptical morphologies is a hollow, prolate, ellipsoidal shell (e.g., Masson 1989). Models of this kind have been widely used to interpret optical and radio observations of $\mathrm{PNe}$ (Masson 1989; Aaquist \& Kwok 1996), including earlier studies of NGC 7027 (e.g., Atherton et al. 1979; Roelfsema et al. 1991; Masson 1989). The high resolution, velocity resolved observations presented here provide new information which we use to evaluate the usefulness and limitations of this picture. 
A simple, ellipsoidal model is characterized by the radii of the principal axes $(a$ and $b$ ), the inclination of the symmetry axis to the line of sight $(i)$, the shell thickness $(t)$, and the velocity field, which we assume to be self-similar (i.e., $V \propto r$ ). We focus on the $\operatorname{Br} \gamma$ observations because they have the best signal-to-noise ratio. In the general case, the density and thickness of the shell are independent, but Masson (1989) has pointed out that they are jointly constrained by the requirement of ionization equilibrium in an ionization bounded PN like NGC 7027. We include this constraint by adopting a constant shell thickness, with an emissivity that varies as $r^{-2}$. The equator of the shell is therefore brighter than the poles.

We have calculated a series of ellipsoidal shell models with different parameters and have used them to best match the $\mathrm{Br} \gamma$ observations. We include in our calculations the instrumental profiles and the important effect of the thermal broadening of the atoms. For H I, the line width at a temperature of $14500 \mathrm{~K}$ appropriate to NGC 7027 (Masson 1989) is $25.8 \mathrm{~km} \mathrm{~s}^{-1}$ (FWHM) in agreement with the measured thermal broadening of the $\mathrm{Br} \gamma$ line (Sect. 3.1). This large line width (which is comparable to the expansion velocity of the shell) accounts for the partially filled-in appearance of the $\operatorname{Br} \gamma$ strip maps (Fig. 4). As discussed by Masson (1989), the geometry of an ellipsoidal model is directly constrained by the emission of the ionized gas. The extent and thickness along the minor axis yield the parameters $b$ and $t$, and the extent of the major axis and the center/limb contrast jointly constrain the axial ratio $a / b$ and the inclination angle $i$.

The best-fit, ellipsoidal model for the $\operatorname{Br} \gamma$ emission has parameters $a=2^{\prime \prime} .8, b=6{ }^{\prime \prime} 4, t=1{ }^{\prime \prime} 1, i=35^{\circ}$, and $v=20 \mathrm{~km} \mathrm{~s}^{-1}$ along the line of sight. The results of this model are compared with the observations in Fig. 8, and can be seen to reproduce some of the gross features of the observational data. These include the approximate shape and the center-limb contrast of the integrated intensity image, as well as the changes in structure and relative intensity at different velocities in the channel and strip maps.

In fitting the observations, the integrated image (top panel) constrains the geometry fairly well: for example changing the inclination by more than $\pm 10^{\circ}$ produces a less satisfactory overall fit. The inclination is further constrained by the velocity resolved data: for large inclination angles the brighter waist of the ellipsoid comes closer to the line of sight through the center of the nebula, and produces brighter emission at the projected expansion velocity, which is not observed. Overall, the shell parameters found here are consistent with previous determinations. In particular, the inclination angle to the line of sight of $35^{\circ}$ is in good agreement with previous estimates of $34^{\circ}$ (Atherton et al. 1979), $40^{\circ}$ (Roelfsema et al. 1991), and $30^{\circ}$ (Scott 1973; Masson 1989).

Despite a general similarity of the ellipsoidal model with the observations, there are important differences which bear on the physical development of the ionized nebula. First the observed emission at low intensity levels
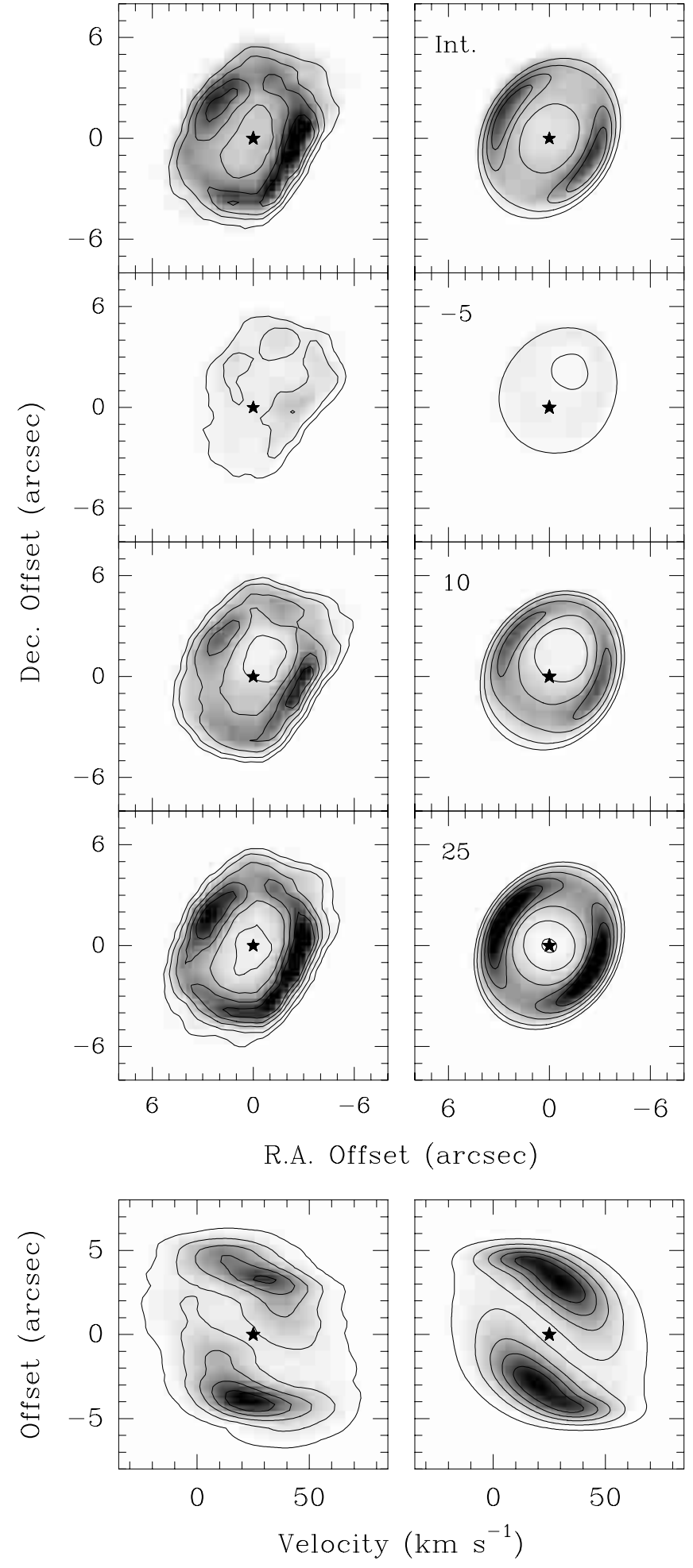

Fig. 8. Comparison of ellipsoidal model of the ionized gas in $\mathrm{Br} \gamma$ with observations. From the top, the panels are: integrated intensity images, channel maps (with the velocity indicated in $\mathrm{km} \mathrm{s}^{-1}$ ), and strip maps along the major axis. The model results are on the right. See text for details. The contour steps are $20 \%$ of representative peaks of the data, with an additional contour at $10 \%$ in the strip maps.

is broader than the model calculations, which reflects the fact that the nebula is not homogeneous, but is composed 

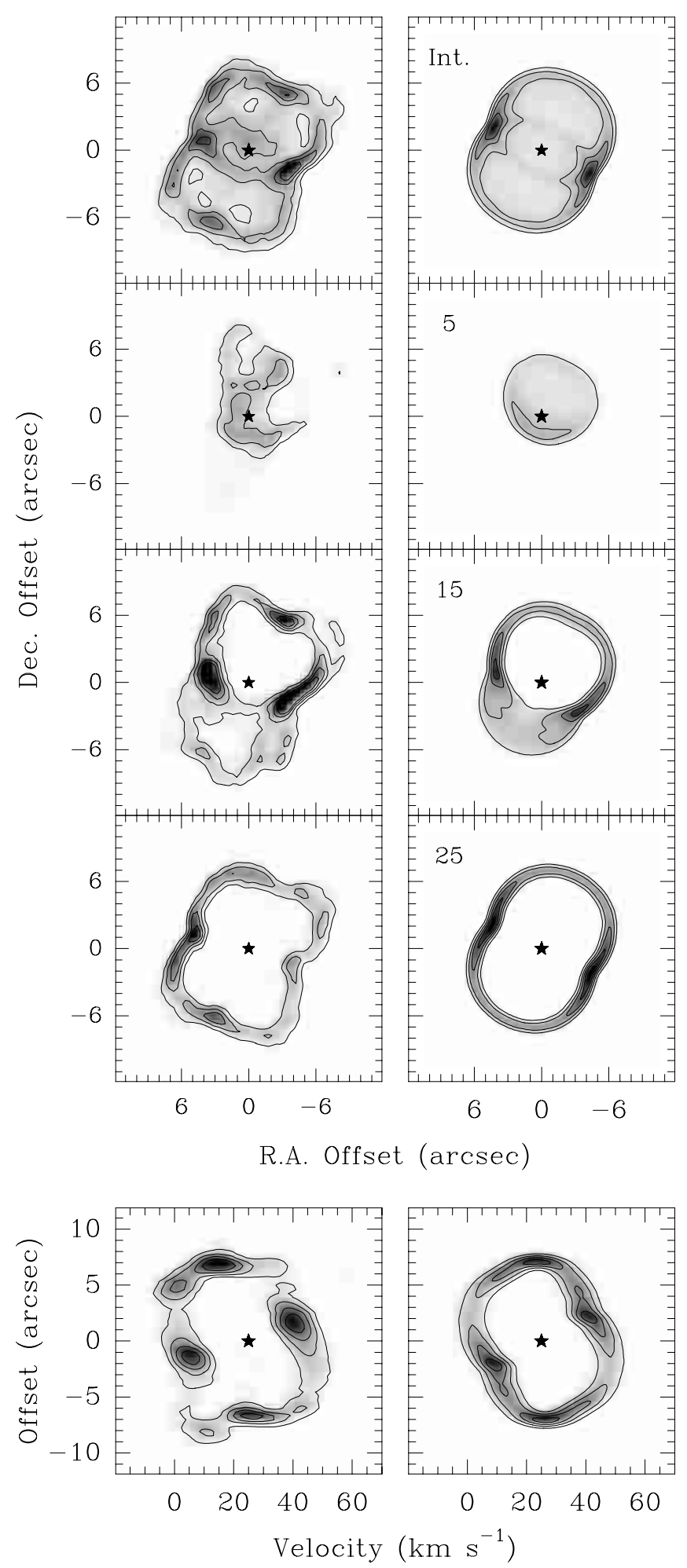

Fig. 9. Comparison of axisymmetric model of the $\mathrm{H}_{2}$ emission with observations. From the top the panels are: integrated intensity images, channel maps (with the velocity indicated in $\mathrm{km} \mathrm{s}^{-1}$ ), and strip maps along the major axis. The model results are on the right. See text for details. The contour steps are $20 \%$ of representative peaks of the data.

of clumps embedded in a more diffuse shell, as already remarked in Sect. 3.1. Second, the observed nebula exhibits nearly parallel sides and flattened ends in both the integrated intensity images and in the channel maps, unlike the more rounded contours of the ellipsoid, i.e., the structure is more cylindrical - as already pointed out by Scott (1973). Third, the model does not account for the highest velocity Br $\gamma$ emission which lies along a bipolar axis offset from the symmetry axis.

All these effects are inconsistent with a smoothly developing ellipsoidal shell, which might be produced in the traditional two-wind model, in which an isotropic fast wind impinges on a slower wind with a smooth density gradient that varies with latitude. They are, however, consistent with a different scenario dominated by directed flows, as discussed further in Sect. 5 .

\subsection{The $\mathrm{H}_{2}$ emission}

We model the $\mathrm{H}_{2}$ emission in NGC 7027 as a thin-walled, hour glass structure as suggested by the appearance of the integrated $\mathrm{H}_{2}$ image and the channel map at the systemic velocity. This kind of geometry is indeed expected in nebulae with a dense equatorial ring and/or high velocity gas flowing predominantly along the polar directions (see, e.g., Matt et al. 2000) and has been used by Latter et al. (2000) to model the spatial and kinematical distribution of $\mathrm{H}_{2}$ emission in NGC 7027. The structure is closed at the ends, and we identify the waist of the hour glass with the ellipse of enhanced emission that surrounds the central star in the $\mathrm{H}_{2}$ image. If we assume the waist to be circular, the shape of the ellipse constrains the inclination of the axis to the line of sight to be $i \sim 60^{\circ}$.

The hour glass shape of the model is represented by a simple analytic function that roughly approximates the cross section seen in the channel map at $v_{\text {sys }}$ and matches the dimensions of the integrated image. The radius of the waist $\left(r_{\mathrm{o}}\right)$ is $4^{\prime \prime} .5$, and the radius of the shell, relative to the waist $\left(r / r_{\mathrm{o}}\right)$, is taken to be $1+0.5 \sin \theta+0.13 \theta \sin ^{2} 2 \theta$, where $\theta$ is the angle of latitude. Thus the axial ratio is 1.5 . The thickness of the shell is taken to be constant $\left(0 .{ }^{\prime \prime} 6\right)$ with the emissivity varying as $r^{-2}$ from the central star. According to PDR models (see Sect. 6), the $\mathrm{H}_{2}$ emission is expected to depend on the incident radiation field and the density, so both these may contribute to the observed variations, as well as limb brightening. For the kinematics, two velocity laws have been examined: a constant radial velocity $\left(20 \mathrm{~km} \mathrm{~s}^{-1}\right)$, and one where $V \propto r$. The second law appears to provide a closer match to the kinematic data, and the results of this model are compared to the observations in Fig. 9 in the form of the velocity-integrated $\mathrm{H}_{2}$ emission, a series of channel maps, and the positionvelocity map along the major axis.

The adopted model reproduces the main characteristics of the observed $\mathrm{H}_{2}$ emission reasonably well. The integrated intensity image (Fig. 9, top panel) is dominated by limb-brightening where the hour glass structure is seen tangentially along the line of sight. In the channel maps at the systemic velocity $\left(v=25 \mathrm{~km} \mathrm{~s}^{-1}\right)$, the $\mathrm{H}_{2}$ emission 
traces a cross section of the hour glass, with the emission strongest at the equator and roughly constant elsewhere. Slight indentations in the $\mathrm{H}_{2}$ emission at the equator are also similar to those observed. At intermediate, blueshifted velocities (the channel map at $v=15 \mathrm{~km} \mathrm{~s}^{-1}$ ), the $\mathrm{H}_{2}$ emission shows a ring-like distribution to the NorthEast, with the southern part of this ring extending along the equator just south of the star, as observed. Extended emission to the south corresponds to the front face of the lower section of the hour glass. At more extreme velocities $\left(v=5 \mathrm{kms}^{-1}\right)$, the extended emission to the north-east corresponds to the front surface of the upper section of the hour glass. The main features of the position-velocity diagram given by this model are also comparable to the observations, in particular the waist at the equator (Fig. 9).

Other inclination angles reproduce the $\mathrm{H}_{2}$ observations less convincingly. For example, as the inclination angle decreases, the equatorial ellipse at the waist becomes more open than observed, and the extreme red/blue-shifted velocities the $\mathrm{H}_{2}$ move too close to the line of sight towards the center. As the angle increases, the equatorial ellipse becomes narrower than observed, and the north and south lobes in each channel map become more alike, and for an inclination of $90^{\circ}$ become identical. We note that Robberto et al. (1993) derived a high value for the inclination angle, i.e. $75^{\circ}$, based on the equatorial ring traced in their $[\mathrm{O} \mathrm{III}] / \mathrm{H} \alpha$ ratio map, although within the uncertainties it is probably consistent with our estimate.

The geometry of our simplified model clearly has limitations in describing the detailed structure seen in the observations. In both the velocity integrated image and at the systemic velocity (Fig. 9), the observations show that the waist is more accented than the model, and it is probable that limb brightening due to the local curvature enhances the waist emission. The ends of the hour glass are also distinctly flatter than the model and show evidence for indentation at the poles, while at offset velocities the ends develop into lobe structures. Our discussion in the next section shows that these distortions are related to the presence of directed flows.

The model in which the expansion velocity is constant is less successful in reproducing some of the main features of the observations. For instance, with $V$ constant, the maximum velocity is along the line of sight to the central star, whereas the observations show that maximum velocity is offset to the north and south for the blue and red-shifted channels, respectively. Also for the model with constant $V$, the enhanced emission at the waist forms convex corners in the major axis strip map which are not seen in the observations. For similar reasons, the model for the $\mathrm{H}_{2}$ emission proposed by Latter et al. (2000), with $V$ constant and an inclination angle of $i=45^{\circ}$, does not provide a good match to the observations. However, given the limitations of our geometrical model, some intermediate law cannot be ruled out.

We note that the inclination angle we estimate for the $\mathrm{H}_{2}$ hour glass differs from that of the ionized gas by $25^{\circ}$. Given the idealized models that underly these estimates, the precise value of the difference is not well constrained. However, in the context of the development of these structures discussed below, a difference in the orientation of the main structures of the ionized gas and $\mathrm{H}_{2}$ could be present, and there are well documented precedents where this is the case, e.g., $\mathrm{BD}+30^{\circ} 3639$ (Bachiller et al. 2000).

\section{Multiple outflows and holes}

Although the most prominent features in our data are consistent with structures that are roughly axi-symmetric, the most striking features in both the ionized and $\mathrm{H}_{2}$ gas deviate from axi-symmetry.

In the $\mathrm{H}_{2}$ channel maps we identify a remarkable series of holes or cavities which are point symmetric about the central star in position and in velocity with respect to $v_{\text {sys }}$. These occur at position angles and velocities of $-53^{\circ} \pm 15 \mathrm{kms}^{-1}, 4^{\circ} \pm 20 \mathrm{~km} \mathrm{~s}^{-1}$, and $-28^{\circ} \pm 25 \mathrm{~km} \mathrm{~s}^{-1}$. They are identified in Fig. 10 and labelled 1, 2 and 3, respectively. The first of these (Outflow 1) is the most prominent, and can also be seen in the underlying integrated intensity $\mathrm{H}_{2}$ image in Fig. 10 - see also the HST image in Latter et al. (2000). Outflow 3 lies close to the major axis, and can also be seen in the $\mathrm{H}_{2}$ major-axis strip map in Fig. 4.

The most direct interpretation of these point symmetric holes is the action of multiple, bipolar outflows or jets from the central star. These are seen to be increasingly common in PNe, and well studied examples where multiple outflows show interactions with the surrounding molecular envelope include CRL 2688 (Cox et al. 2000) and M1-16 (Huggins et al. 2000). In both of these cases the jets are more prominent than in NGC 7027, but the similarity is striking.

The high velocity gas seen in the $\operatorname{Br} \gamma$ line is clearly a related phenomenon. At velocities $\gtrsim 25 \mathrm{~km} \mathrm{~s}^{-1}$ from the systemic velocity the $\operatorname{Br} \gamma$ emission shows a change in direction from rough alignment to the major axis, to a position angle of $\sim 55^{\circ}$. This direction is coincident with the most prominent holes along the axis of Outflow 1, as shown in the upper panels of Fig. 10. It also aligns with distinct minima in the $\mathrm{CO}$ emission in the surrounding envelope (Fig. 7).

As shown in Sect. 4.2, the kinematics of both the ionized and $\mathrm{H}_{2}$ in NGC 7027 are consistent with a homologous expansion, i.e. $V \propto r$. If this is the case, the velocity axes in the BEAR data cubes corresponds to a space axis, and allows us to study the $3-\mathrm{D}$ structure of the ionized region and the PDR. Views of the $\mathrm{H}_{2}$ and $\mathrm{Br} \gamma$ data cubes which make use of 3-D visualization techniques are shown in Fig. 11. In this representation, a surface contour has been constructed in the data cubes at $20 \%$ of the peak emission, and the front views of the surface contours are shown in the figure, illuminated obliquely from the rear with directed and diffuse light. These panels resemble the maps in Figs. 2 and 5 in outline, but the 3-D structure of the different parts of the molecular envelope and the 

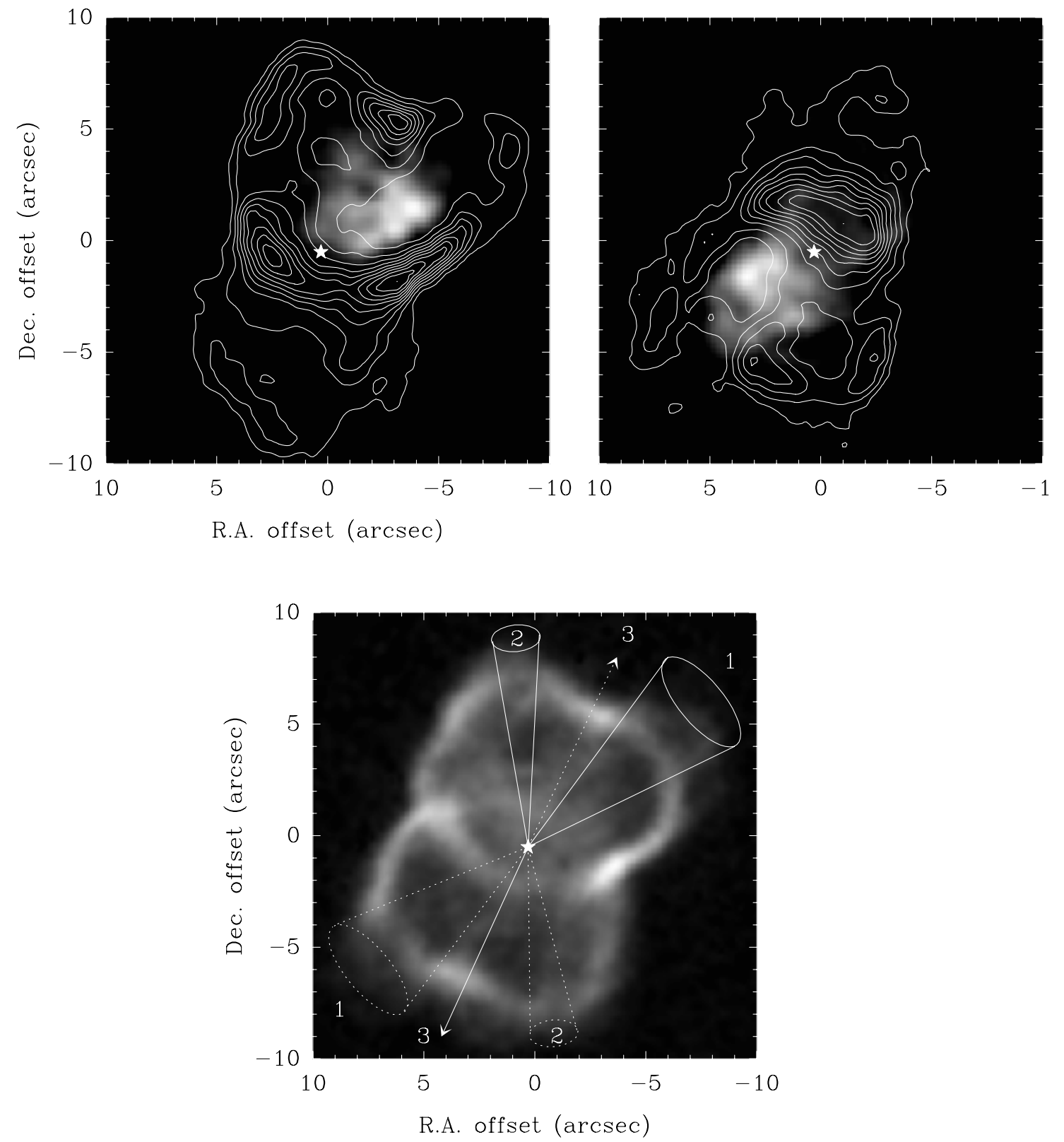

Fig. 10. Identification of the outflow axes in NGC 7027. The bottom panel shows the three outflow axes identified in the $\mathrm{H}_{2}$ channel maps, superposed on the velocity integrated $\mathrm{H}_{2}$ emission. The outflow axis labelled 1 can also be identified in the integrated image. The top panel shows the $\mathrm{H}_{2}$ channel maps (contours) at intermediate velocities $\left(10\right.$ and $40 \mathrm{~km} \mathrm{~s}^{-1}$ in the left and right panels, respectively) superposed on the extreme velocity channels of the $\operatorname{Br} \gamma$ emission $\left(-30\right.$ and $80 \mathrm{~km} \mathrm{~s}{ }^{-1}$ respectively).

ionized region are clearly seen, in particular the series of holes or cavities.

The overall structure of the $\mathrm{H}_{2}$ emission is an envelope with a series of openings above and below the equator. The equator itself is a relatively complete structure with a distinct waist, as described in Sect. 3.2. There are also dents in the polar caps. The $3-\mathrm{D}$ representation underscores the remarkable symmetries in the global structure of the $\mathrm{H}_{2}$ envelope. In contrast, the ionized region has a relatively smooth distribution, and the $3-\mathrm{D}$ view shows clearly the elongated shell structure with the openings towards the poles. The comparison of the $\mathrm{H}_{2}$ and $\mathrm{Br} \gamma$ 3 -D images underlines the difference in the orientations between the ionized and molecular gas which was noted in Sect. 4.3.

The geometry of the $\mathrm{H}_{2}$ features that we observe could have been formed by simultaneous, multiple outflows or by a single, episodic bipolar outflow that wobbles or precesses in direction. In either case the small size of the cavities, which subtend angles of $\approx 10^{\circ}-25^{\circ}$ at the central star, indicate that the outflows are fairly narrowly collimated. The expansion time scale of the $\mathrm{H}_{2}$ emitting gas of $\approx 1500$ yr likely sets an upper limit on the age of the outflows, but they could be much more recent. The presence of the high velocity $\mathrm{Br} \gamma$ only along the axis of Outflow 1 (Fig. 10) suggests that this is the youngest outflow, which 

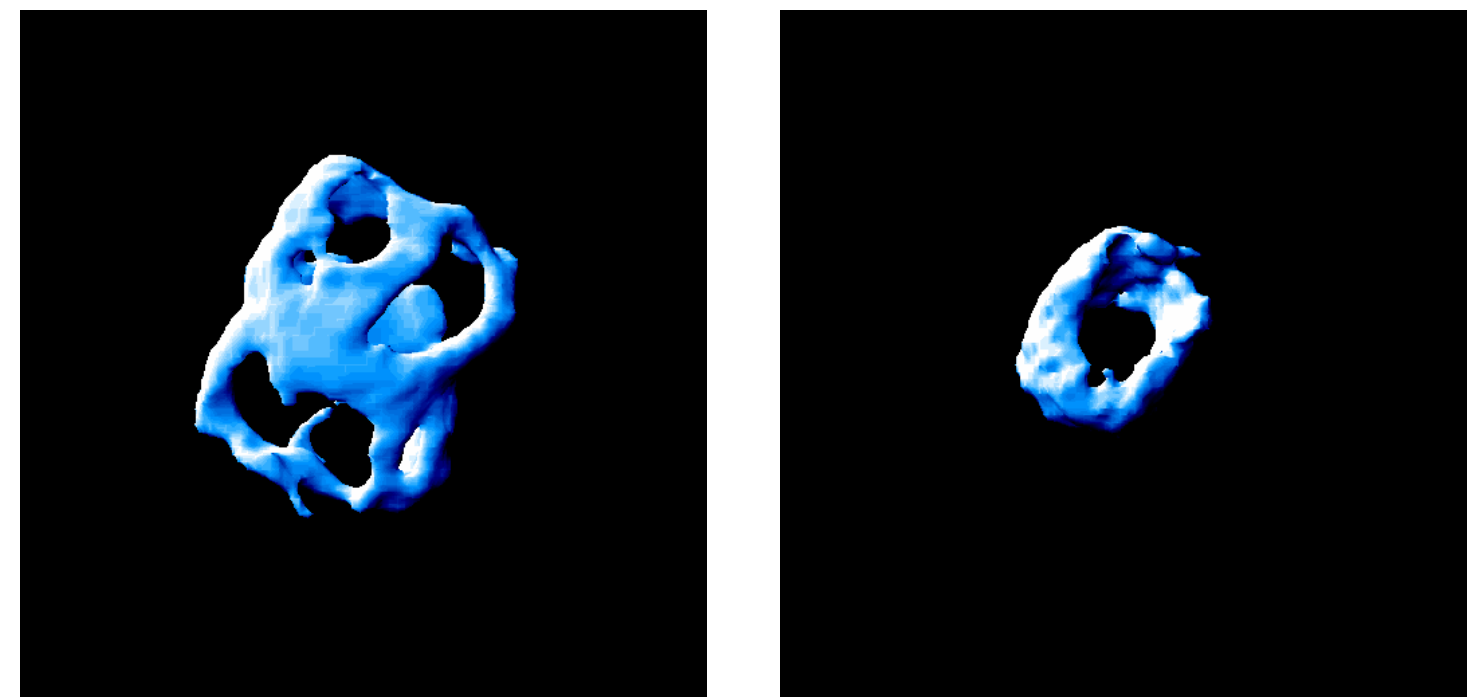

Fig. 11. Three-dimensional representations of the $\mathrm{H}_{2} 1-0 \mathrm{~S}(1)$ and $\mathrm{Br} \gamma$ emission in NGC 7027 (left and right panels). The views show surface contours of the $\mathrm{H}_{2}$ and $\mathrm{Br} \gamma$ emission as seen from the front, i.e. as observed.

would favor the wobbling or precessing model. In this context it is interesting to note that recent observations with the Chandra X-ray Observatory have discovered extended X-ray emission in NGC 7027 (Kastner et al. 2001). The $\mathrm{X}$-ray emission is aligned with the high-velocity $\mathrm{Br} \gamma$ emission (Fig. 12). There is a close correspondence between them which is best seen in the northwest where the nebula is tilted towards the observer and where the X-ray emission is less absorbed than in the southeast. It has been suggested that the X-ray emission from NGC 7027 is the result of a past or still active fast wind from the central star (Kastner et al. 2001) colliding with the slower expanding envelope. The high temperature involved $\left(T \sim 3 \times 10^{6} \mathrm{~K}\right)$ implies wind velocities of $\sim 400 \mathrm{~km} \mathrm{~s}^{-1}$. The high-velocity ionized gas detected in the $\operatorname{Br} \gamma$ emission would need to be close to the plane of the sky to be compatible with the wind velocities required to explain the X-ray emission. In this case, the event would have happened less than 100 years ago, if one adopts a mean distance of the fast $\operatorname{Br} \gamma$ wind of $\sim 7^{\prime \prime}$ from the centre of the nebula.

\section{The photodissociation region of NGC 7027}

In addition to information on the morphology and kinematics of NGC 7027, the present data provide new constrains on the physical conditions in the inner molecular envelope. Previous near-infrared studies have shown that the $\mathrm{H}_{2}$ emission lines in NGC 7027 are consistent with excitation in dense, UV excited gas (Graham et al. 1993b; Cox et al. 1996; Yan et al. 1999; Latter et al. 2000). The clear inside-outside stratification of the $\mathrm{H}_{2}$ and $\mathrm{CO}$ emission seen in Fig. 7 is precisely that expected in a PDR, adding further support to the above excitation scenario. Shock excitation is unlikely to play a dominant role (Cox et al. 1996) although some regions could be locally excited by shocks (for instance along the north-west/south-east outflow axis).

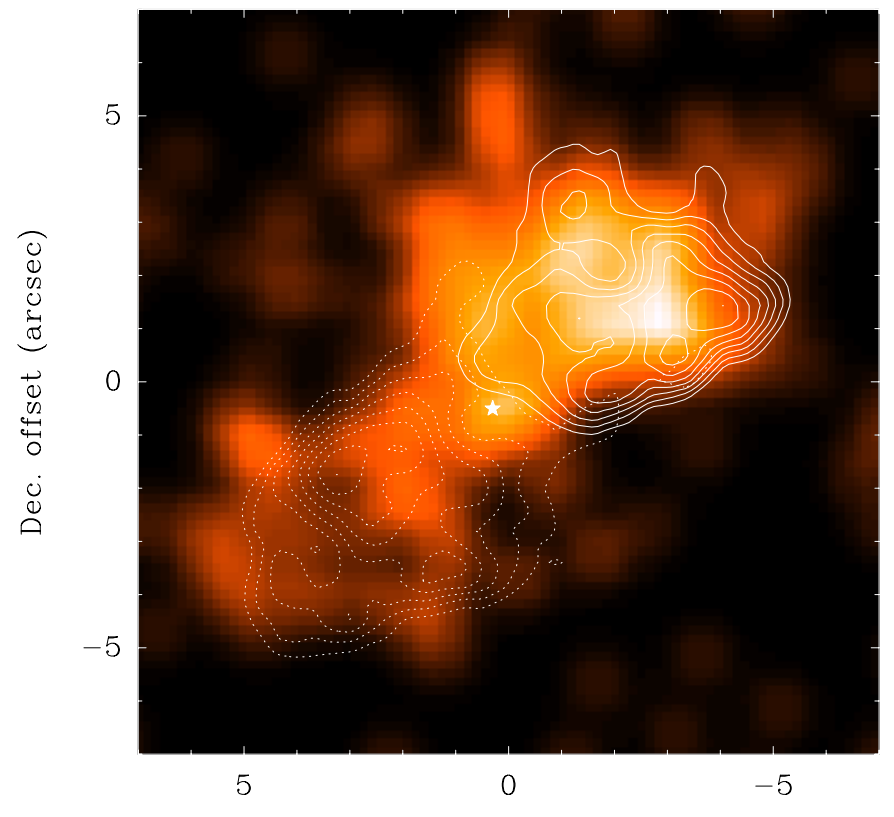

R.A. offset (arcsec)

Fig. 12. Overlay of high velocity $\operatorname{Br} \gamma$ emission with the Chandra X-ray Observatory image of NGC 7027. The X-ray data (from Kastner et al. 2001) is shown as a grey scale. The $\operatorname{Br} \gamma$ emission, from channels at -30 and $+80 \mathrm{~km} \mathrm{~s}^{-1}$ is shown as full and dashed contours, respectively.

One of the new aspects of the present data is the detailed information on the spatial distribution of the velocity resolved $\mathrm{H}_{2}$ emission in NGC 7027. It is thus interesting to compare the observed structure of the transition regions in the $\mathrm{PDR}$ (through the emission of $\mathrm{H}_{2}$ and $\mathrm{CO}$ at the systemic velocity which minimizes the line of sight effects) with predictions of PDR models. In the following, we describe such a comparison based on the stationary PDR model of Le Bourlot et al. (1993). The PDR is 


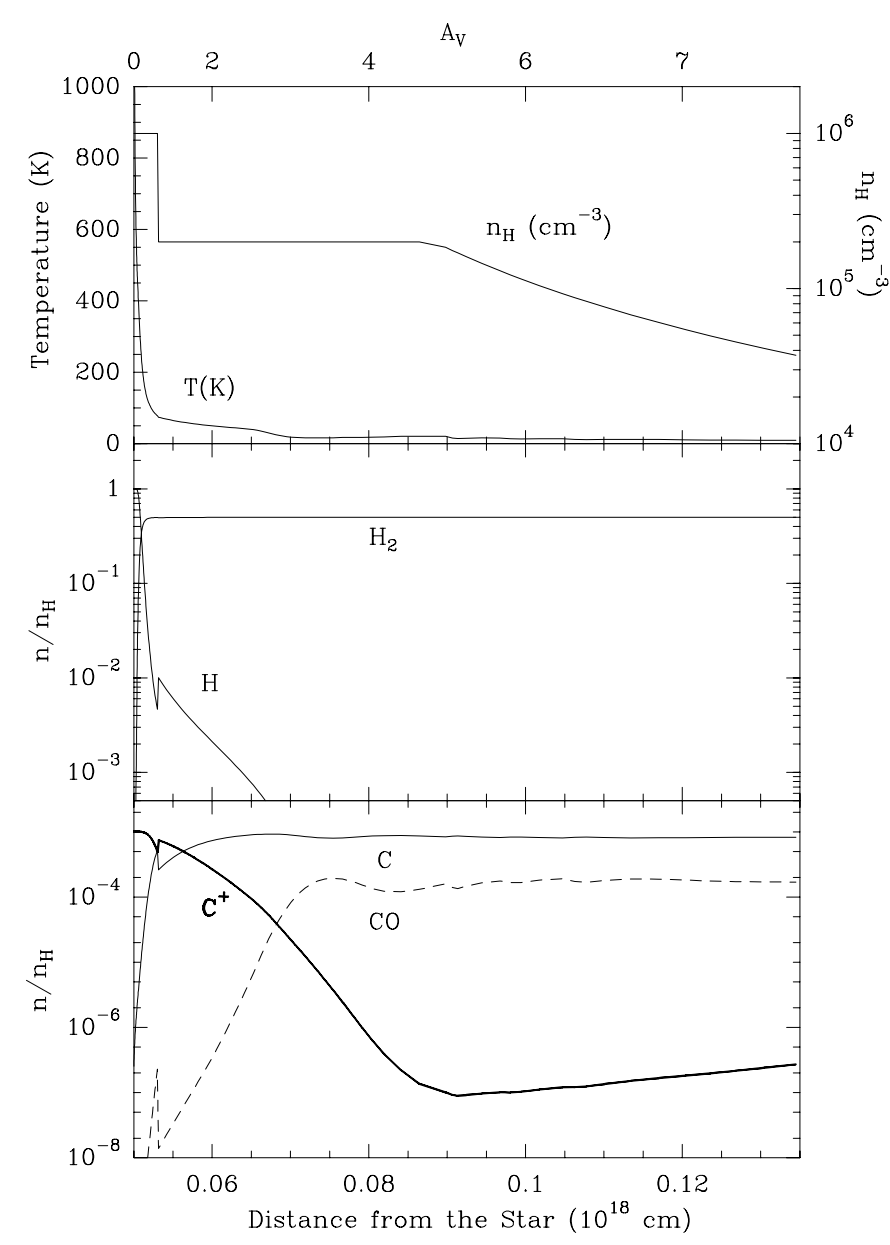

Fig. 13. Results of the PDR model for NGC 7027. The top panel shows the profiles of gas temperature $(T)$ and densities $\left(n_{\mathrm{H}}\right)$ as a function of distance from the central star (in $\mathrm{cm}$ and visual extinction $A_{\mathrm{v}}$ ). The stellar light is assumed to impinge with no extinction on the PDR's surface at $5 \times 10^{16} \mathrm{~cm}$. The two other panels show the abundance profiles of the major atomic and molecular species across the PDR - further details are given in the text.

represented by a semi-infinite, plane parallel slab with an incident radiation field on the interface. Starting from the interface, the radiative transfer, the gas thermal balance and the chemical balance are solved for each step into the slab. The two main input parameters of the model are $\chi$, the scaling factor for the FUV radiation field, and $n_{\mathrm{H}}$, the gas proton density. We adopted the gas phase elemental abundances from Middlemass (1990) with C, O, and $N$ abundances by number of $10 \times 10^{-4}, 4.3 \times 10^{-4}$ and $1.9 \times 10^{-4}$, respectively.

The far-UV radiation incident upon the neutral envelope (at a distance of $\theta=3.4^{\prime \prime}$ or $4.5 \times 10^{16} \mathrm{~cm}$ from the star) is estimated to be $\chi \approx 3 \times 10^{4}$ in units of the mean Solar Neighborhood radiation (given in $1.6 \times$ $10^{-3} \mathrm{erg} \mathrm{cm}^{-2} \mathrm{~s}^{-1}$ ) for the adopted distance of $880 \mathrm{pc}$ and a central star luminosity and temperature of $L=$ $7.7 \times 10^{3} L_{\odot}$ and $T=198000 \mathrm{~K}$ (Latter et al. 2000). The density distribution in the PDR was chosen so as to reproduce the observed spatial distributions of the $\mathrm{H}_{2}$ and

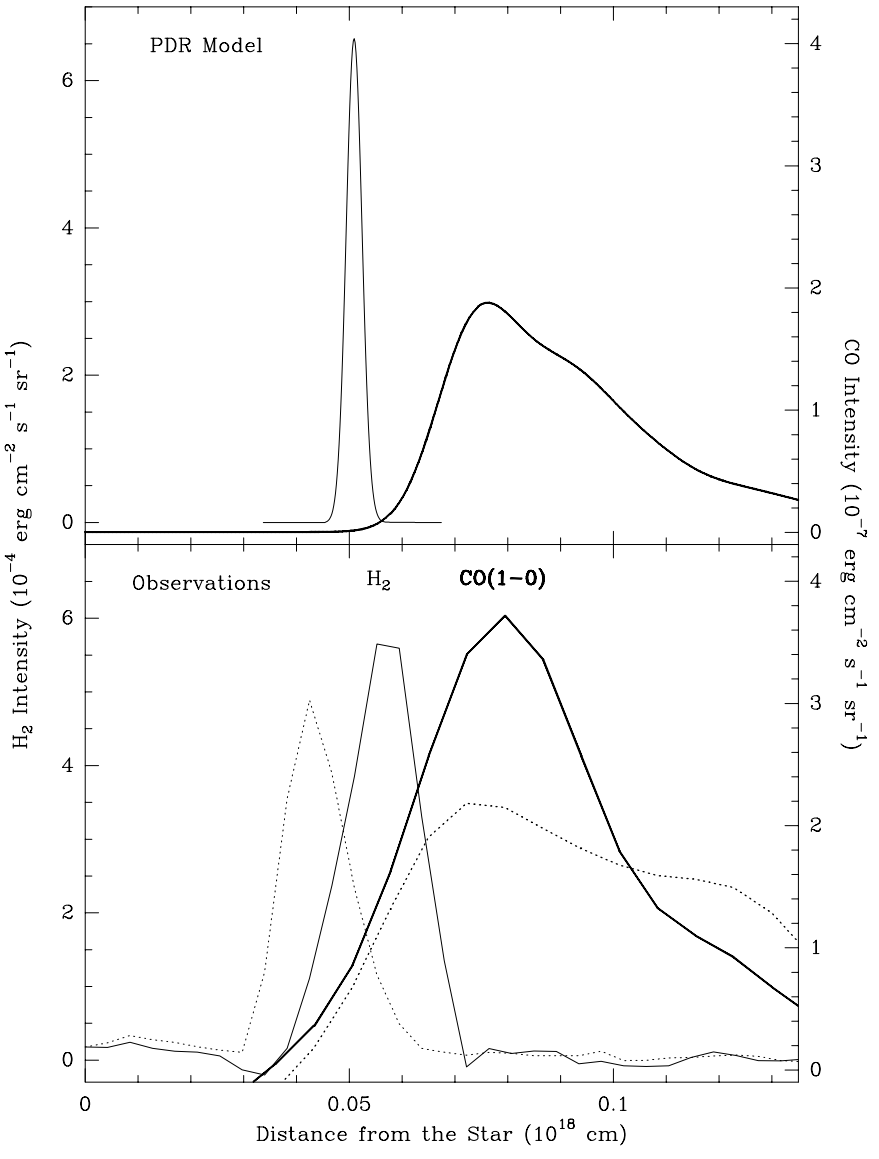

Fig. 14. Bottom panel: observed distribution along the minor axis of the emissions at the systemic velocity of $25 \mathrm{~km} \mathrm{~s}^{-1}$ of $\mathrm{CO}(1-0)$ (from Graham et al. 1993b), $\mathrm{H}_{2}$ 1-0 S(1) and $\mathrm{Br} \gamma$ (from this paper). The east and west sides are shown as full and dashed lines, respectively. The distance is given with respect to the central star (the distance scale has been derived for an adopted distance of $880 \mathrm{pc}$ to NGC 7027). Top panel: results of the PDR model calculations for $\mathrm{H}_{2}$ 1-0 S(1) and $\mathrm{CO}(1-0)-$ see text for details.

CO emission. The best fit to the observations was found for a density distribution with three layers: a dense and thin layer, a layer with a constant density and an outer region where the density decreases as the inverse-square of the distance:

$n_{\mathrm{H}}(r)=\left\{\begin{array}{lrl}n_{\mathrm{H}}^{0} & : & r<r_{0} \\ n_{\mathrm{H}}^{1} & : & r_{0}<r<r_{1}, \\ n_{\mathrm{H}}^{1} \times\left(r / r_{1}\right)^{-2}: & r>r_{1}\end{array}\right.$

where $n_{\mathrm{H}}^{0}=10^{6} \mathrm{~cm}^{-3}$ is the density in the $r<r_{0}$ region with $r_{0}=3 \times 10^{15} \mathrm{~cm}$, and $n_{\mathrm{H}}^{1}=2 \times 10^{5} \mathrm{~cm}^{-3}$ is the density in the $r_{0}<r<r_{1}$ region with $r_{1}=4 \times 10^{16} \mathrm{~cm}$. The top panel of Fig. 13 shows the adopted density profile together with the corresponding temperature structure. The resulting distributions across the PDR of the relative abundances of $\mathrm{H}$ and $\mathrm{H}_{2}$, and of $\mathrm{C}^{+}, \mathrm{C}$ and $\mathrm{CO}$ are shown in the middle and bottom panels as a function of the distance from the exciting star.

In Fig. 14, the predicted spatial stratifications and intensities of the 1-0 S(1) $\mathrm{H}_{2}$ and the $\mathrm{CO}(1-0)$ line emissions 
are compared to the observed distributions and line intensities. These cuts along the minor axis have been obtained from the images centered at the systemic velocity $\left(v_{\text {sys }} \sim 25 \mathrm{~km} \mathrm{~s}^{-1}\right)$ integrated over a velocity width $\left(\approx 6 \mathrm{~km} \mathrm{~s}^{-1}\right)$ corresponding to slices in the plane of the sky. These cuts represent the nebular structure with a minimum line of sight effect and can therefore be compared with the model predictions on the morphology and the line intensities. The model results presented in Fig. 14 have been integrated along the line of sight over a width corresponding to $\approx 3 \times 10^{16} \mathrm{~cm}$. The model results have been convolved with the instrumental profile of the $\mathrm{H}_{2}$ observations ( $F H W M$ of 0 .' 6$)$ and the synthesized beamwidth of $2.8^{\prime \prime}$ of the $\mathrm{CO}(1-0)$ observations. Under these assumptions, the observed spatial stratification and the line emission are well matched by the PDR model. The predicted position of the $\mathrm{CO}$ emission agrees with the observed position (with a peak at a distance of $\approx 7.5 \times 10^{16} \mathrm{~cm}$ ) and the predicted width $\left(F W H M\right.$ of $\left.5.5 \times 10^{15} \mathrm{~cm}\right)$ is consistent with the observations. Note that the observed extension of the $\mathrm{CO}(1-0)$ emission is also predicted by the model. For the 1-0 S(1) $\mathrm{H}_{2}$ emission, the adopted parameters of the PDR model predict a position of the peak emission which is also close to the observed position, at a distance of $5 \times 10^{16} \mathrm{~cm}$ from the star, and a width of $4.5 \times 10^{15} \mathrm{~cm}$ which is similar to the width observed with $B E A R$ or the HST.

From the model of the $\mathrm{H}_{2}$ kinematics (Sect. 4.3), the velocity gradient is $2.6 \mathrm{~km} \mathrm{~s}^{-1}$ per $10^{16} \mathrm{~cm}$, so that the geometrical depth of the slice corresponding to $v_{\text {sys }} \pm$ $3 \mathrm{~km} \mathrm{~s}^{-1}$ is $2.3 \times 10^{16} \mathrm{~cm}$, i.e. a fraction of the inner radius of the PDR. This is comparable to the column needed to explain the observed intensities with an homogeneous, plane parallel PDR model. The differences between the model predictions and the observations could reflect the complex structure of the nebula seen in the $\mathrm{H}_{2}$ emission as a series of small-scale, clumpy features (Sect. 3.2 and the HST image) which are not taken into account by the simple model. Also the distribution of the gas is not symmetric as shown by the differences in the profiles in the east and west parts of the nebula (Fig. 14).

The present PDR model is slightly different from previous models used to describe NGC 7027. To reproduce the high excitation CO emission lines (Liu et al. 1996) and rotational transitions of $\mathrm{CH}^{+}$(Cernicharo et al. 1997), Yan et al. (1999) have modeled the neutral envelope of NGC 7027 with a thin $\left(3 \times 10^{15} \mathrm{~cm}\right)$, high-density $\left(2 \times 10^{6} \mathrm{~cm}^{-3}\right)$, inner shell, in addition to the usual stellar wind envelope. Whereas this model reproduces the observed lines intensities of the $\mathrm{CO}$ and $\mathrm{CH}^{+}$rotational lines and $\mathrm{H}_{2}$ lines, it does not reproduce the observed spatial stratification which is described here. In particular, the model by Yan et al. does not reproduce the position and the extension of the $\mathrm{CO}$ emission. A plateau of relatively dense gas between the thin, high-density layer and the outflow region is needed to explain the $\mathrm{CO}$ distribution (Fig. 13). Clearly further observations and theoretical work is needed to explore the physical conditions (temperature and density distributions) of the PDR associated with NGC 7027. Further comparison of the present PDR model with all available observations on NGC 7027 is deferred to a future paper (Habart et al. in preparation).

In any case, the fact that the intensity of the 1-0 S(1) $\mathrm{H}_{2}$ line emission can be roughly explained by a simple PDR model suggests that the excitation of $\mathrm{H}_{2}$ by UV photons is the dominant process in NGC 7027. If at all, the excitation by X-rays or shocks could be important locally such as in the outflow regions of NGC 7027 described in Sect. 5.

\section{Conclusions}

The near-infrared spectro-imaging presented in this paper shows the detailed kinematics and the complex morphology of the ionized gas and the surrounding molecular envelope in the young PN NGC 7027. Due to the high spatial and spectral resolution of the BEAR data, new features are revealed that underscore the role of multiple outflows during the early evolution of PNe.

Major differences are seen in the morphology of the ionized gas and the $\mathrm{H}_{2}$ emission in NGC 7027. The ionized gas forms an elongated, limb-brightened shell with nearly parallel sides and distinctly flattened ends, and the brightness is patchy, indicating the presence of numerous small condensations. The $\mathrm{H}_{2}$ emission is distributed at the periphery of the ionized cavity and appears as a limbbrightened, bi-conical shell, with enhanced emission at the equator. The $\mathrm{H}_{2}$ emission also traces the inner boundary of the extended molecular envelope, consistent with expectations for the excitation of $\mathrm{H}_{2}$ in a thin photo-dissociation region. Both the intensities and the spatial stratification of the $1-0 \mathrm{~S}(1) \mathrm{H}_{2}$ and $\mathrm{CO}(1-0)$ line emissions are well explained by a stationary PDR model.

Simple axi-symmetric models of the observations constrain the geometry of NGC 7027. Modeling the ionized gas as an expanding, ellipsoidal shell and the $\mathrm{H}_{2}$ emission as a thin-walled, hour glass structure with a radial velocity field $(V \propto r)$ reproduces some of the main features of the observational data. Both structures are clearly tilted to the line of sight, towards Earth to the north west, although the inclination angles based on these models differ by about $25^{\circ}$. However, these simple models do not reproduce the many inhomogeneities seen in the data, nor do they account for the striking deviations from axi-symmetry which are observed in both the ionized and $\mathrm{H}_{2}$ gas.

In the ionized gas, $\mathrm{Br} \gamma$ emission is detected at high velocities, along a bipolar axis that is not aligned with the major axis of the nebula. In $\mathrm{H}_{2}$, there is a remarkable series of lobes and holes at the ends of the hour glass structure that are point symmetric about the center. These structures indicate a recent, dramatic history of bipolar outflows from the center of NGC 7027, which have pierced and disrupted the remnant AGB envelope, inducing irreversible structural changes which are now seen as the $\mathrm{H}_{2}$ lobes and holes. The high-velocity $\mathrm{Br} \gamma$ emission 
aligns with high temperature X-ray emission, and likely traces the most recent $(\approx 100 \mathrm{yr})$ fast wind activity.

Recent observations of other young PNe provide additional examples with unambiguous evidence for envelopeoutflow interactions. These include: KjPn 8 where powerful optical jets have excavated biconical cavities in the surrounding molecular envelope (Forveille et al. 1998); $\mathrm{BD}+30^{\circ} 3639$ where symmetric, high-velocity $\left(50 \mathrm{~km} \mathrm{~s}^{-1}\right)$ molecular bullets have been detected where the jets impact the surrounding neutral gas (Bachiller et al. 2000); and M1-16 where the outflows have excavated large bipolar cavities and created symmetric lobes of molecular gas by entrainment (Huggins et al. 2000). Even in the case of the fully developed Helix nebula, the molecular gas of the envelope has preserved the effects of the impacts of the outflows (Young et al. 1999). Finally, it should be noted that complex, multi-polar structures are also seen in the ionized gas of essentially all young PNe (e.g., Sahai \& Trauger 1998). All these examples, together with the case of NGC 7027, suggest that the change in mass-loss mode and the resulting envelope-outflow interactions must be a common phenomenon resulting from a basic mechanism in the stellar evolution of stars of a few solar masses at the end of the AGB phase.

Acknowledgements. We thank J. R. Graham for sending us his CO data cube of NGC 7027 and J. H. Kastner the Chandra X-ray image. We are grateful to G. Pineau des Forêts for valuable discussions on photodissociation regions. We thank D. Mekarnia for his help during part of the data reduction. This work was supported in part by NSF grant AST-9986159 (to PJH).

\section{References}

Aaquist, O. B., \& Kwok, S. 1990, A\&AS, 84, 229

Atherton, P. D., Hicks, T. R., Reay, N. K., et al. 1979, ApJ, 232,786

Bachiller, R., Forveille, T., Huggins, P. J., Cox, P., \& Maillard, J.-P. 2000, A\&A, 353, L5

Bieging, J. H., Wilner, D., \& Thronson, H. A. Jr. 1991, ApJ, 379,271

Bryce, M., Pedlar, A., Muxlow, T., Thomasson, P., \& Mellema, G. 1997, MNRAS, 284, 815

Cernicharo, J., Liu, X.-W., González-Alfonso, et al. 1997, ApJ, 483, L65

Cox, P., Maillard, J.-P., Huggins, P. J., et al. 1996, A\&A, 293, 871

Cox, P., Lucas, R., Huggins, P. J., et al. 2000, A\&A, 353, L25

Dinerstein, H. L. 2001, ApJ, 550, L223

Elias, J. H., Frogel, J. A., Mathews, K., \& Neugebauer, G. 1992, AJ, 87, 1029
Forveille, T., Huggins, P. J., Bachiller, R., \& Cox, P. 1998, ApJ, 495, L111

Geballe, T. R., Burton, M. G., \& Isaacman, R. 1991, MNRAS, 253,75

Graham, J. R., Herbst, T., Matthews, K., et al. 1993a, ApJ, 408, L105

Graham, J. R., Serabyn, E., Herbst, T., et al. 1993b, AJ, 105, 250

Hajian, A. R., Terzian, Y., \& Bignell, C. 1993, AJ, 106, 1965

Huggins, P. J., Bachiller, R., Cox, P., \& Forveille, T. 1996, A\&A, 315, 284

Huggins, P. J., Forveille, T., Bachiller, R., \& Cox, P. 2000, ApJ, 544, 889

Kaler, J. B., Aller, L. H., Epps, H. W., \& Czyzak, S. J. 1976, ApJSS, 31, 163

Kastner, J. H., Gatley, I., Merrill, K. M., Probst, R., \& Weintraub, D. A. 1994, ApJ, 421, 600

Kastner, J. H., Vrtilek, S. D., \& Soker, N. 2001, ApJ, 550, L189

Latter, W. B., Dayal, A., Bieging, J. H., et al. 2000, ApJ, 539, 783

Le Bourlot, J., Pineau des Forêts, G., Roueff, E., \& Flower, D. R. 1993, A\&A, 267, 233

Liu, X.-W., Barlow, M. J., Nguyer-Q-Rieu, et al. 1996, A\&A, 315, L257

Luhman, K. L., \& Rieke, G. H. 1996, ApJ, 461, 298

Maillard, J. P., \& Michel, G., 1982, in Instrumentation for Astronomy with Large Telescopes, ed. C. M. Humphries (D. Reidel), IAU Colloq., 92, 213

Maillard, J.-P. 1995, in 3-D Optical spectroscopy methods in astronomy, ed. G. Comte, \& M. Marcellin, IAU Colloq. 149, ASP Conf. Ser., 71, 316

Maillard, J. P. 2000, in Imaging the Universe in 3 Dimensions, ed. E. van Breughel, \& J. Bland-Hawthorn, ASP Conf. Ser., 195,185

Masson, C. R. 1989, ApJ, 336, 294

Matt, S., Balick, B., Winglee, R., \& Goodson, A. 2000, ApJ, 545,965

Middlemass, D. 1990, MNRAS, 244, 294

Péquignot, D., \& Baluteau, J.-P. 1994, A\&A, 283, 593

Robberto, M., Clampin, M., Ligori, S., Paresce, F., \& Staude, H. J. 1993, A\&A, 280, 241

Roelfsema, P. R., Goss, W. M., Pottasch, S. R., \& Zijlstra, A. 1991, A\&A, 251, 611

Sahai, R., \& Trauger, J. T. 1998, AJ, 116, 1357

Scott, P. F. 1973, MNRAS, 161, 35

Simons, D. A., Clark, C. C., Smith, S., et al. 1994, in Instrumentation in Astronomy VIII, Proc. SPIE, 2198, 185

Smith, H. A., Larson, H. P., \& Fink, U. 1981, ApJ, 244, 835

Woodward, C. E., Pipher, J. L., Forrest, W. J., Moneti, A., \& Shure, M. A. 1992, ApJ, 385, 567

Yan, M., Federman, S. R., Dalgarno, A., \& Bjorkman, J. E. 1999, ApJ, 515, 640

Young, K., Cox, P., Huggins, P. J., Forveille, T., \& Bachiller, R. 1999, ApJ, 522, 387 